\title{
Reciprocal stabilization of ABL and TAZ regulates osteoblastogenesis through transcription factor RUNX2
}

\author{
Yoshinori Matsumoto, ${ }^{1}$ Jose La Rose, ${ }^{1}$ Oliver A. Kent, ${ }^{1}$ Melany J. Wagner, ${ }^{1}$ Masahiro Narimatsu, ${ }^{2}$ Aaron D. Levy, ${ }^{3}$ \\ Mitchell H. Omar, ${ }^{3}$ Jiefei Tong, ${ }^{4}$ Jonathan R. Krieger, ${ }^{4}$ Emily Riggs, ${ }^{5}$ Yaryna Storozhuk, ${ }^{6}$ Julia Pasquale, ${ }^{6}$ Manuela Ventura, \\ Behzad Yeganeh, ${ }^{8}$ Martin Post, ${ }^{8}$ Michael F. Moran, ${ }^{4}$ Marc D. Grynpas, ${ }^{6}$ Jeffrey L. Wrana, ${ }^{2}$ Giulio Superti-Furga, ${ }^{9}$ \\ Anthony J. Koleske, ${ }^{3}$ Ann Marie Pendergast, ${ }^{5}$ and Robert Rottapel ${ }^{1,10,11,12,13}$ \\ 1Princess Margaret Cancer Center, University Health Network, University of Toronto, Toronto, Ontario, Canada. ${ }^{2}$ Samuel Lunenfeld Research Institute, Mount Sinai Hospital, Toronto, Ontario, Canada. \\ ${ }^{3}$ Department of Molecular Biophysics and Biochemistry, Yale University, New Haven, Connecticut, USA. Program in Molecular Structure and Function, Hospital for Sick Children, Toronto, Ontario, Canada. \\ ${ }^{5}$ Department of Pharmacology and Cancer Biology, Duke University School of Medicine, Durham, North Carolina, USA. ${ }^{6}$ Lunenfeld-Tanenbaum Research Institute, Mount Sinai Hospital, Toronto, Ontario, \\ Canada. ${ }^{7}$ TECHNA Institute for the Advancement of Technology for Health, University Health Network, Toronto, Ontario, Canada. ${ }^{8}$ Program in Physiology and Experimental Medicine, Hospital for Sick \\ Children, Toronto, Ontario, Canada. ${ }^{9}$ CeMM Research Center for Molecular Medicine of the Austrian Academy of Sciences, Vienna, Austria. ${ }^{10}$ Department of Medicine, "Department of Medical Biophysics, and \\ ${ }^{12}$ Department of Immunology, University of Toronto, Toronto, Ontario, Canada. ${ }^{13}$ Division of Rheumatology, St. Michael's Hospital, Toronto, Ontario, Canada.
}

\begin{abstract}
Cellular identity in metazoan organisms is frequently established through lineage-specifying transcription factors, which control their own expression through transcriptional positive feedback, while antagonizing the developmental networks of competing lineages. Here, we have uncovered a distinct positive feedback loop that arises from the reciprocal stabilization of the tyrosine kinase ABL and the transcriptional coactivator TAZ. Moreover, we determined that this loop is required for osteoblast differentiation and embryonic skeletal formation. ABL potentiated the assembly and activation of the RUNX2TAZ master transcription factor complex that is required for osteoblastogenesis, while antagonizing PPAR $\gamma$-mediated adipogenesis. ABL also enhanced TAZ nuclear localization and the formation of the TAZ-TEAD complex that is required for osteoblast expansion. Last, we have provided genetic data showing that regulation of the ABL-TAZ amplification loop lies downstream of the adaptor protein 3BP2, which is mutated in the craniofacial dysmorphia syndrome cherubism. Our study demonstrates an interplay between ABL and TAZ that controls the mesenchymal maturation program toward the osteoblast lineage and is mechanistically distinct from the established model of lineage-specific maturation.
\end{abstract}

\section{Introduction}

Cellular identity in multicellular organisms is frequently established through lineage-specifying transcription factors that control their own expression, usually through transcriptional positive feedback (1-4), while antagonizing the expression of factors that control the commitment of competing lineages (5).

Mesenchymal multipotential progenitor cells can differentiate into specialized cell lineages including osteoblasts, adipocytes, chondrocytes, and myocytes. Transcriptional coactivator with the PDZ-binding motif (TAZ), a paralog of YAP, is a key transcriptional coactivator for transcriptional enhancer-associated domain-mediated (TEAD-mediated) cell proliferation (6-9). TAZ has also been shown to be required for mesenchymal lineage commitment (10). TAZ and RUNX2 (also known as core-binding factor 1 [CBFA1]) form a transcriptional complex that drives develop-

Note regarding evaluation of this manuscript: Manuscripts authored by scientists associated with Duke University, The University of North Carolina at Chapel Hill, Duke-NUS, and the Sanford-Burnham Medical Research Institute are handled not by members of the editorial board but rather by the science editors, who consult with selected external editors and reviewers.

Conflict of interest: The authors have declared that no conflict of interest exists. Submitted: March 29, 2016; Accepted: September 22, 2016.

Reference information: / Clin Invest. 2016;126(12):4482-4496. doi:10.1172/JCI87802. ment of the osteoblast lineage, while TAZ coordinately represses $\operatorname{PPAR} \gamma$-dependent gene transcription, which is important for adipocyte lineage commitment (10). RUNX2 is the master transcription factor for osteoblast differentiation, as mice lacking RUNX2 fail to undergo bone ossification as a result of defective osteoblast differentiation $(11,12)$. However, the regulation of the RUNX2TAZ complex during osteoblastogenesis remains unclear.

Mesenchymal commitment toward the osteoblast lineage is essential for embryonic skeletal development (13). Mineralized bones are synthesized by osteoblasts through 2 different processes: chondrocyte-independent (intramembranous) and chondrocyte-dependent (endochondral) bone formation (13). Intramembranous osteoblasts in the calvarium and clavicles differentiate directly from mesenchymal progenitor cells, whereas longitudinal endochondral bone growth requires both osteoblasts and chondrocytes within the cartilaginous-rich growth plates (13). Both processes are required for embryonic skeletal formation.

We have previously studied cherubism, a rare hereditary syndrome associated with severe craniofacial developmental defects in children $(14,15)$. Cherubism arises from missense mutations in the SH3BP2 gene, which stabilize the steady-state protein levels of the adapter protein SH3-domain binding protein 2 (3BP2) (14-16). 3BP2, which was originally identified as a binding protein for the ABL kinase (abelson murine leukemia viral oncogene 
homolog 1) (17, 18), is essential for normal bone formation, as Sh $3 b p 2^{-1-}$ mice display osteoporosis due to defective osteoblastogenesis (19). We have demonstrated that endogenous ABL kinase is activated by $3 \mathrm{BP} 2$ in osteoblasts and that the ectopic expression of $\mathrm{ABL}$ rescued the defective mineralization observed in 3BP2-depleted osteoblasts (19). Consistent with our data showing that $3 \mathrm{BP} 2$ and $\mathrm{ABL}$ are part of a common genetic and bio-

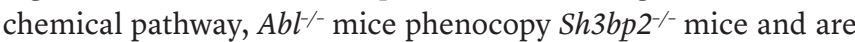
severely osteoporotic due to an osteoblast defect (20). However, the molecular mechanism by which the activation of ABL by 3BP2 regulates bone formation is obscure.

In distinction to the well-established model that transcriptional networks control the lineage-specific maturation program in multicellular organisms, we have uncovered a protein amplification feedback loop between ABL and TAZ that is required for osteoblastogenesis and embryonic skeletal formation. This study points to a link between the 3BP2/ABL-signaling pathway and the RUNX2TAZ transcriptional complex during osteoblast maturation.

\section{Results}

$A B L$ is required for osteoblast expansion, differentiation, and embryonic skeletal development. To investigate the developmental function of ABL within the osteoblast compartment, we analyzed embryonic bone formation in $\mathrm{Abl}^{-/-}$newborn pups and observed abnormal boney structures (Figure 1A). $A b l^{-/-}$pups displayed impaired intramembranous ossification with hypomineralization of the calvarium and enlarged anterior and posterior fontanelles (Figure 1B and Supplemental Figure 1A; supplemental material available online with this article; doi:10.1172/JCI87802DS1). Other skeletal structures including clavicles, ribs, and limb bones were hypoplastic in $\mathrm{Abl}^{-/-}$pups (Figure 1C and Supplemental Figure 1B). Femurs from $\mathrm{Abl}^{-/-}$newborn pups showed a $48 \%$ loss of total bone volume (Supplemental Figure 1C) as a consequence of decreased femur length and cortical bone thickness compared with WT pups (Supplemental Figure 1, B and C). Trichrome staining of tibiae from $\mathrm{Abl}^{-/}$pups revealed severe trabecular bone loss compared with WT pups (Figure 1D). Histomorphometric analysis of tibiae from $\mathrm{Abl}^{-/}$pups showed reduced osteoblast numbers per bone surface and trabecular bone volume per total volume, indicating an intrinsic osteoblast defect (Figure 1E). Furthermore, TRAP staining of tibiae showed that the ratios of osteoclast surface to bone surface and osteoclast numbers to bone surface were similar in WT and $\mathrm{Abl}^{-/}$pups (Supplemental Figure 1, D and E). These results demonstrate that $\mathrm{ABL}$ is required for normal bone formation, which involves both intramembranous and endochondral ossification during embryonic skeletal development.

To investigate whether ABL kinase activity is required for enhancing osteoblast maturation, we used an FKBP chimeric form of ABL, whose activity is enhanced by the small molecule FK1012 (21). In osteoblast progenitor cells, we observed that kinase-active FKBP-ABL (WT), but not kinase-dead FKBP-ABL (KD), was sufficient to trigger osteoblast mineralization (Figure $1 F)$. Importantly, FKBP-ABL (WT) potentiated the expression of bone $\gamma$-carboxyglutamate protein (Bglap) mRNA, a transcriptional marker of osteoblast differentiation, whereas cells expressing FKBP-ABL (KD) failed to induce Bglap expression (Figure 1G). Expression of FKBP-ABL (WT) in Saos-2 cells cultured in growth medium with FK1012 demonstrated that active ABL was sufficient to bypass the requirement of osteogenic medium to induce mineralization and BGLAP transcription (Supplemental Figure 1, F and $G)$. Last, we observed that cell expansion and BrdU incorporation were reduced in ABL-depleted primary murine osteoblasts (Figure 1, $\mathrm{H}$ and I, and Supplemental Figure 1, H and I), demonstrating that $\mathrm{ABL}$ is also required for optimal osteoblast proliferation. These data show that ABL is required for skeletal development through a program that involves osteoblast expansion, differentiation, and mineralization.

Active ABL assembles the RUNX2-TAZ transcription factor complex required for osteoblast differentiation. Next, we investigated the molecular mechanism by which ABL regulates embryonic bone formation. We observed that endogenous protein levels of $\mathrm{ABL}$ and tyrosine phosphorylation content were increased during osteoblast differentiation in primary murine calvarial cells (Figure 2A). Our observation that the defective mineralization and skeletal development associated with $\mathrm{Abl}^{-/-}$newborn pups phenocopies the Run $x 2^{+/-}$heterozygous newborns $(13,22)$ suggested a potential genetic link between ABL and RUNX2. Previous work, which demonstrated that RUNX2 and TAZ form a transcription factor complex required for BGLAP transcription (10), prompted us to query whether ABL could induce Bglap transcription through the RUNX2-TAZ complex. We observed that a constitutively active form of ABL (P242E/P249E, ABL [PP]), but not a kinase-dead version of ABL (K290M, ABL [KD]) (23), increased the RUNX2-TAZ complex formation by 8 -fold in $293 \mathrm{~T}$ cells (Figure 2B), whereas treatment with the ABL kinase inhibitor imatinib reduced the complex formation of these proteins (Supplemental Figure 2A). Similarly, the induction of active FKBP-ABL (WT) was able to enhance the interaction of endogenous RUNX2 and TAZ proteins in Saos-2 cells (Supplemental Figure 2B). These results corresponded to a 3-fold increase in the BGLAP transcriptional activity of RUNX2 and TAZ in the presence of active ABL (Figure $2 \mathrm{C}$ ), indicating that active $A B L$ enhances osteoblastogenesis through the formation of the RUNX2-TAZ complex.

We next queried whether ABL was a direct component of the RUNX2-TAZ complex and showed by coprecipitation that ABL bound to both RUNX2 and TAZ in a kinase-dependent manner, either in 2 binary complexes or as part of a single ternary complex (Figure 2, D and E). To determine whether ABL functions as a scaffold protein bridging RUNX2 and TAZ, we coprecipitated RUNX2 with TAZ, in which ABL was depleted by shRNA, and observed a loss in the interaction between RUNX2 and TAZ (Figure 2F), with attenuation of RUNX2-TAZ transcriptional activity in 293T cells (Supplemental Figure 2C).

Next, we asked whether formation of the RUNX2-TAZ complex was dependent on tyrosine phosphorylation of either RUNX2 and/or TAZ by ABL and observed that both RUNX2 and TAZ, but not their respective all-tyrosine-to-phenylalaninemutant variants (YF) were abundantly tyrosine phosphorylated when coexpressed with active ABL (PP) (Figure 2, G and $\mathrm{H})$. Moreover, tyrosine phosphorylation levels of endogenous RUNX2 and TAZ were reduced in ABL-depleted primary murine osteoblasts, with loss in the assembly of the RUNX2-TAZ complex and a concomitant loss of the transcriptional activity of this complex (Figure 2, I and J). 

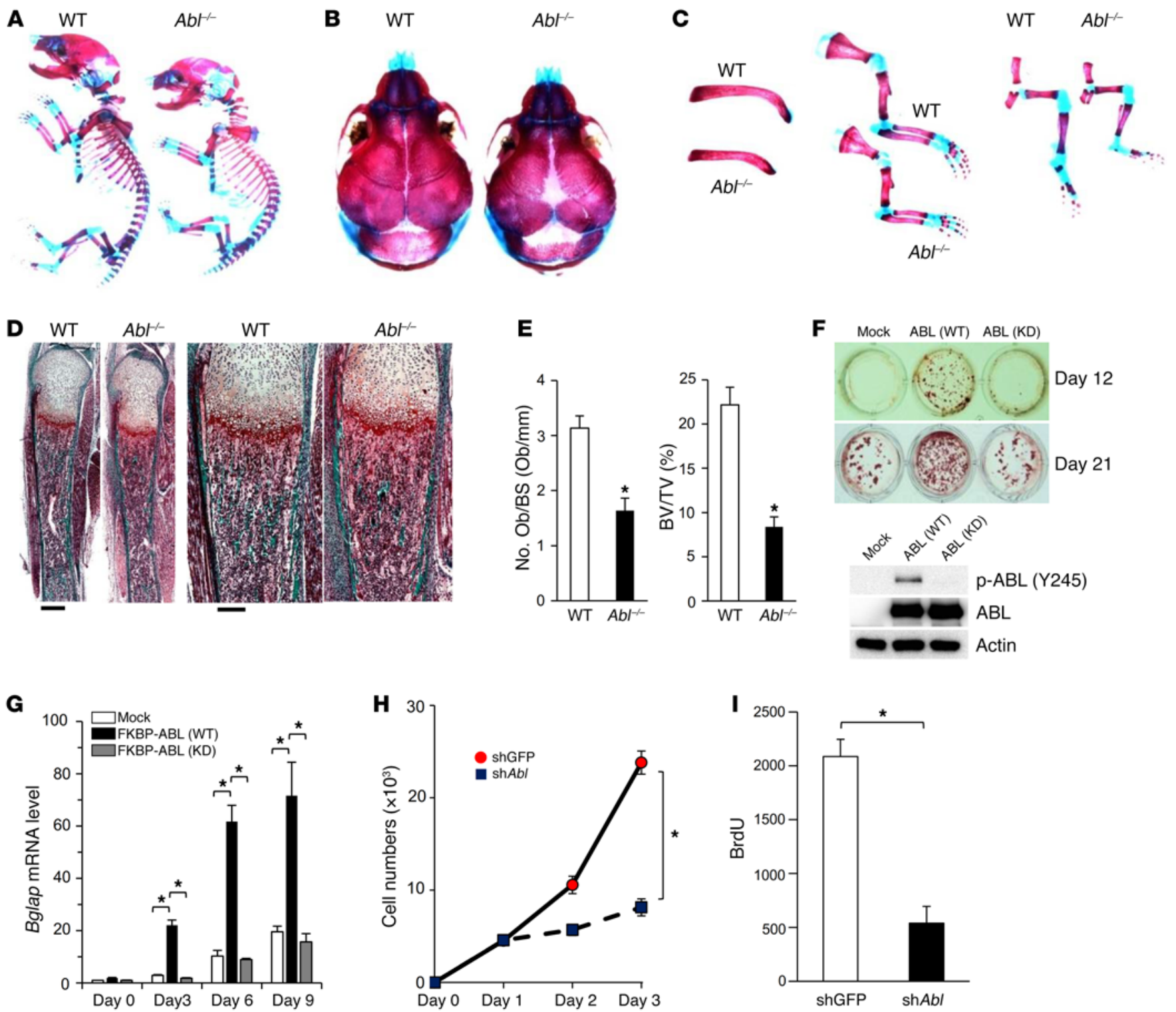

Figure 1. ABL is required for osteoblast expansion, differentiation, and embryonic skeletal development. (A-C) Whole-mount skeletons (A), calvarium (B), clavicles, and limb bones (C) of WT and $A b l-K O\left(A b^{-/-}\right)$newborn pups stained with alizarin red and Alcian blue. (D) Trichrome staining of tibiae from WT and $\mathrm{Abl}^{-1-}$ newborn pups. The calcified tissue appears blue-green. Scale bars: $400 \mu \mathrm{m}$ (left), $200 \mu \mathrm{m}$ (right). (E) Histomorphometric analysis of osteoblast numbers per bone surface (No. Ob/BS) and trabecular bone volume per total volume (BV/TV) in WT and $A b^{-1-}$ newborn pups. $n=5$. (F) Primary murine osteoblasts infected with an empty vector control (Mock) or with a retroviral vector expressing WT FKBP-ABL (WT) or kinase-dead FKBP-ABL (KD) were cultured in osteogenic medium for 12 and 21 days and stained with alizarin red S solution. $n=3$. ABL immune complexes were probed with an antip-ABL (Y245) or anti-ABL antibody. WCLs were probed with an anti-actin antibody as a loading control. (C) qPCR analysis of Bglap mRNA expression in cells in $\mathbf{F}$ cultured in osteogenic medium for 3 to 9 days. $n=3$. (H) Growth curves of primary murine osteoblasts infected with an shRNA targeting GFP (shGFP) or $A b l(s h A b l)$ and cultured for 3 days. $n=3$. (I) Primary murine osteoblasts in $\mathbf{H}$ were cultured in the presence of BrdU for 24 hours. Cells were subjected to fixation within the culture plate, and BrdU incorporation was determined by ELISA. $n=5 .{ }^{*} P<0.05$, by ANOVA with a Tukey-Kramer post-hoc test $(\mathbf{G})$ or by unpaired $t$ test $(\mathbf{E}, \mathbf{H}$, and $\mathbf{I})$. Data represent the mean $\pm \mathrm{SEM}$.

To confirm that tyrosine phosphorylation of RUNX2 and TAZ by $\mathrm{ABL}$ is required for complex formation, we coprecipitated RUNX2 with TAZ and observed that the RUNX2 (YF) mutant poorly formed a complex with TAZ compared with WT RUNX2 and was transcriptionally inactive (Supplemental Figure 2, D and E). Similarly, the TAZ (YF) mutant formed an attenuated complex with RUNX2 compared with WT TAZ, resulting in reduced transcriptional coactivation (Supplemental Figure 2, F and G). These data demonstrate that phosphorylation of both RUNX2 and TAZ was required for the formation of the ternary active transcription factor complex.

Last, we investigated whether the ability of active ABL to bypass osteogenic medium and induce osteoblast mineralization in Saos- 2 cells was contingent on RUNX2 and TAZ expression. We observed that knockdown of RUNX2 or TAZ abolished the mineralization induced by active FKBP-ABL in Saos-2 cells (Figure 2, $\mathrm{K}$ and L, and Supplemental Figure 2, $\mathrm{H}$ and I). These data show 
A

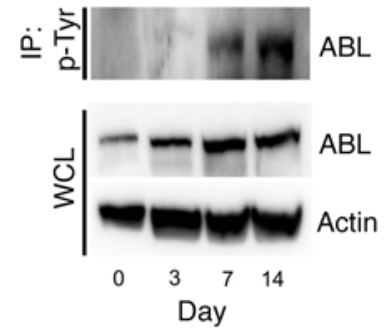

B

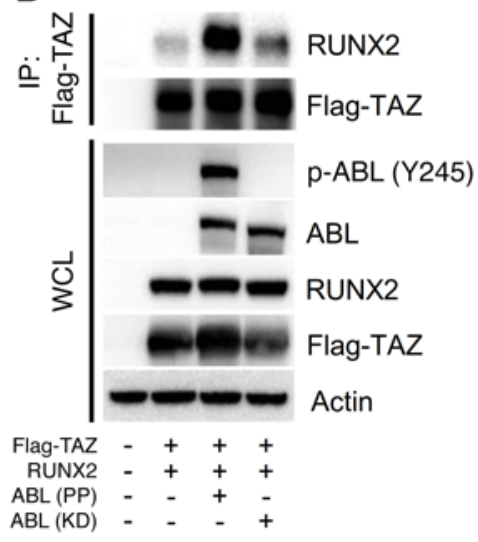

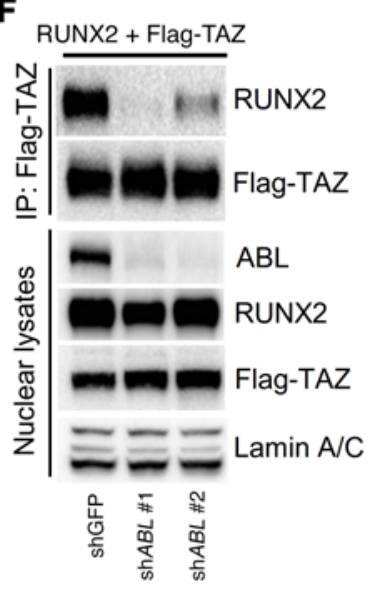

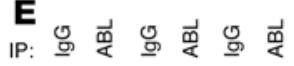

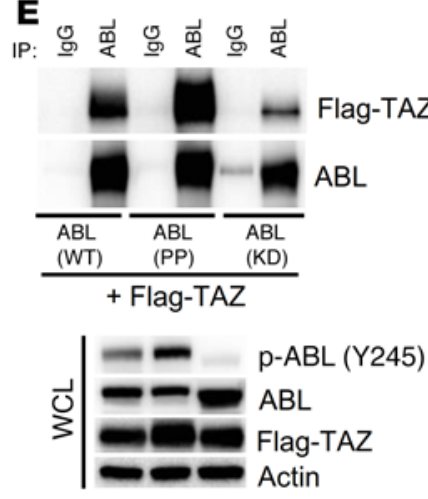

$\begin{array}{ll}\text { Flag-TAZ }++ & + \\ \text { ABL (WT }+- & -\end{array}$

$\mathrm{ABL}(\mathrm{PP})=+-$

$\mathrm{ABL}(\mathrm{KD})$ - -+
C

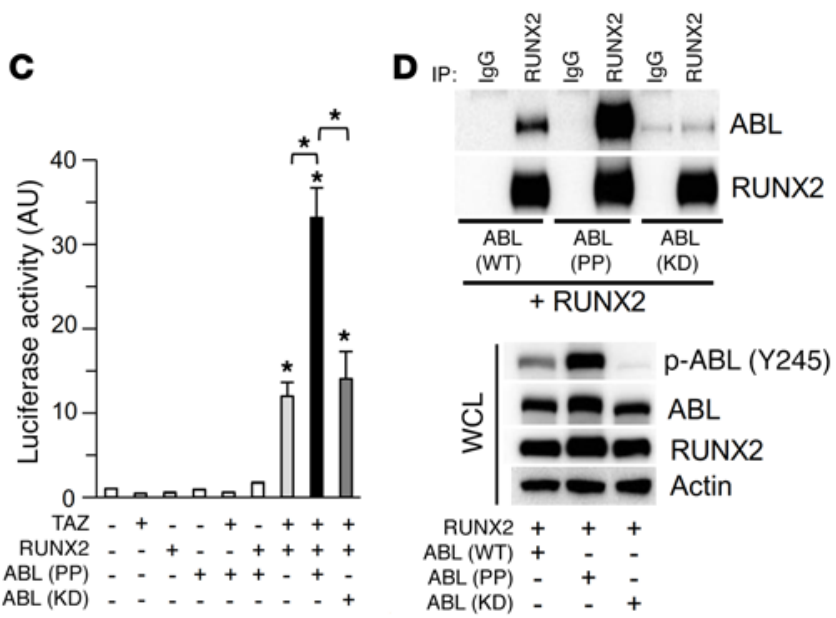

G

H

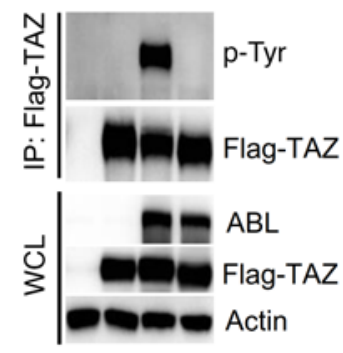

RUNX2 (WT) - + + -

RUNX2 (YF) - - -+

ABL (PP) - - + +
Flag-TAZ (WT) -++

Flag-TAZ (YF) - - -+ $\mathrm{ABL}(\mathrm{PP})$ - - + +

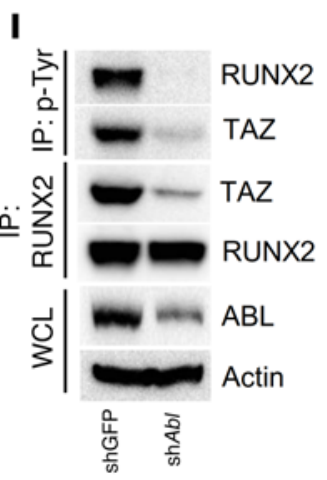

J

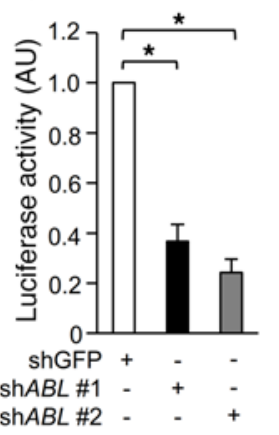

$\mathbf{K}$

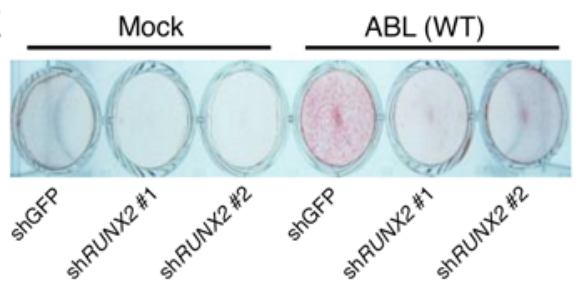

$\mathbf{L}$

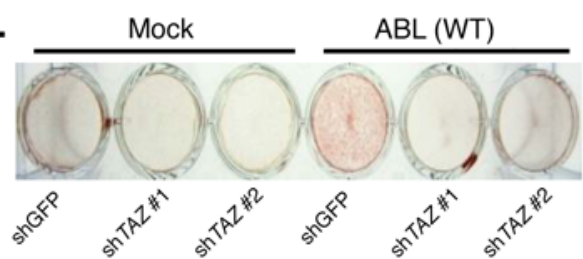

Figure 2. Active ABL assembles the RUNX2-TAZ transcription factor complex required for osteoblast differentiation. (A) Primary murine osteoblasts were cultured in osteogenic medium for 14 days, and phosphotyrosine immune complexes were probed with an anti-ABL antibody. WCLs were probed with the indicated antibodies for Western blot analysis. (B) HEK293T cells were cotransfected with Flag-TAZ and RUNX2, with or without ABL (PP or KD). FlagTAZ immune complexes were probed with an anti-RUNX2 antibody. (C) Luciferase activity from a BGLAP reporter assay in HEK293T cells cotransfected with the indicated constructs. $n=3$. ( $(\mathbf{D}$ and $\mathbf{E})$ HEK293T cells were cotransfected with the indicated constructs, and RUNX2 (D) or ABL (E) immune complexes were probed with an anti-ABL (D) or anti-Flag (E) antibody. (F) HEK293T cells infected with shGFP or shABL were cotransfected with RUNX2 and Flag-TAZ. The nuclear compartment was extracted from the cells, and Flag-TAZ immune complexes were probed with an anti-RUNX2 antibody. (G and $\mathbf{H})$ HEK293T cells were cotransfected with WT or YF RUNX2 (C) or Flag-TAZ (H), with or without ABL (PP). RUNX2 (C) or Flag-TAZ (H) immune complexes were probed with an anti-p-Tyr antibody. (I) Primary murine osteoblasts infected with shGFP or shABL were cultured in osteogenic medium. p-Tyr or RUNX2 immune complexes were probed with an anti-RUNX2 or anti-TAZ antibody. (J) Luciferase activity from a Bglap reporter assay in primary murine osteoblasts in the presence of shGFP or shABL. $n=3$. ( $(\mathbf{K}$ and $\mathbf{L}$ ) Saos-2 cells infected with an empty vector control or an FKBP-ABL-expressing retroviral vector in the presence of shGFP (K and $\mathbf{L}$ ), shRUNX2 (K), or shTAZ (L) were cultured in growth medium containing 50 nM FK1012. Cells were stained with alizarin red $S$ solution. $n=3$. ${ }^{*} P<0.05$, by ANOVA with a Tukey-Kramer post-hoc test. Data represent the mean \pm SEM. 
that ABL scaffolds and phosphorylates the RUNX2-TAZ complex, which is required for the transcriptional activity that drives the osteoblastogenic differentiation program.

Nuclear $A B L$ is required for the transcriptional activity of the RUNX2-TAZ complex. Our findings that ABL interacts with the nuclear transcription factor RUNX2-TAZ complex suggested a nuclear function of ABL during osteoblast maturation. Therefore, we examined the intracellular localization of ABL in murine calvarial cells cultured in either growth medium or osteogenic medium. Under standard growth conditions, ABL was localized exclusively in the cytoplasm, whereas in cells grown in osteogenic medium, a large fraction of total ABL protein accumulated in the nucleus (Figure 3A and Supplemental Figure 3A), showing a correlation between nuclear ABL localization and osteoblast maturation. We then examined the role of each of the individual components of the osteogenic medium and found that ascorbic acid was sufficient to trigger the nuclear translocation of $A B L$, while neither $\beta$-glycerophosphate nor dexamethasone were able to do so (Figure $3 \mathrm{~A}$ ). Additionally, the protein expression levels of TAZ and RUNX2 were increased in the nucleus in osteoblasts cultured in osteogenic medium (Figure 3B). These data support a role for the nuclear ABL-RUNX2-TAZ complex during osteoblast maturation.

To demonstrate that $\mathrm{ABL}$ is a component of a transcriptional complex regulating BGLAP expression, we performed ChIP of the endogenous BGLAP promoter in Saos-2 cells and observed strong enrichment of a BGLAP-derived amplicon in both ABL and RUNX2 chromatin immunoprecipitates (Figure 3C). Moreover, we found that the recruitment of ABL to the BGLAP promoter was enhanced during osteoblast maturation (Supplemental Figure 3B) and was dependent on RUNX2 expression (Figure 3D). Last, to confirm whether nuclear ABL was required for regulation of the RUNX2-TAZ complex, we showed that an ABL mutant lacking its nuclear localization sequence $(\triangle \mathrm{NLS})$, which is localized exclusively in the cytoplasm (24), was unable to potentiate RUNX2-TAZ transcriptional activity (Figure $3 \mathrm{E}$ ). These data show that $\mathrm{ABL}$ is targeted to the nucleus during osteoblastogenesis and recruited to the BGLAP promoter as part of the RUNX2-TAZ complex.

$A B L$ stabilizes TAZ through the suppression of a ubiquitin-mediated degradation pathway. We noted that TAZ protein levels were significantly increased in primary osteoblasts and MC3T3 cells cultured in ascorbic acid, without alteration in Taz transcripts (Figure 4A and Supplemental Figure 4, A and B), suggesting that TAZ protein was probably posttranscriptionally stabilized. To determine whether TAZ protein expression is dependent on ABL, we examined TAZ in ABL-deficient primary calvarial osteoblasts and observed a significant reduction in TAZ protein expression levels, without a reduction in Taz transcript levels (Figure 4B and Supplemental Figure 4C). Consistent with a loss of ABL and reduced TAZ levels, we observed defective mineralization in ABL-deficient calvarial osteoblasts (Supplemental Figure 4D). We investigated whether $\mathrm{ABL}$ kinase activity regulated TAZ protein levels and found that expression of active (PP), but not KD, ABL increased both ectopic and endogenous TAZ protein expression levels in 293T (Figure 4C) and Saos-2 (Supplemental Figure 4E) cells. Pulse-chase measurements demonstrated that active ABL (PP) increased the half-life of TAZ from 5.6 hours to 15.8 hours (Figure 4D), while ABL (PP), but not ABL (KD), suppressed TAZ ubiquitylation (Figure 4E).
YAP has previously been reported to be stabilized following tyrosine phosphorylation by ABL (25). In distinction, we observed that all-tyrosine-to-phenylalanine-mutant TAZ (YF) was also stabilized by active ABL (PP) (Supplemental Figure 4F), suggesting that TAZ stabilization by ABL is not mediated by tyrosine phosphorylation. TAZ is degraded following the phosphorylation of Ser311 by LATS, which primes for the phosphorylation of Ser314 by CK1 1 (26). Phosphorylated Ser314 lies in the TAZ phosphodegron and triggers binding to and subsequent ubiquitylation of TAZ by the E3-ubiquitin ligase $\beta$-TrCP (26). We hypothesized that ABL suppressed TAZ ubiquitylation through disruption of the interaction between TAZ and $\beta$-TrCP and observed that ABL (PP), but not ABL (KD), diminished the interaction between these proteins (Figure $4 \mathrm{~F}$ ). These data demonstrate that ABL stabilizes TAZ through the suppression of its ubiquitin-mediated degradation pathway initiated by $\beta$-TrCP.

$A B L$ stabilizes the TAZ-TEAD complex required for osteoblast expansion. We have shown that ABL mediated stabilization of TAZ protein and asked whether ABL enhances TAZ activity. As shown in Figure 5A, ABL (PP), but not ABL (KD), triggered TAZ nuclear accumulation. Nuclear TAZ interacts with the TEAD transcription factor to induce a cell proliferation transcriptional program (8). We queried whether the proliferation defect observed in ABL-depleted osteoblasts (Figure $1 \mathrm{H}$ ) was mediated through regulation of the TAZ-TEAD complex. We found that ABL (PP), but not ABL (KD), enhanced the formation of the TAZ-TEAD1 complex (Figure 5B) and induced the TEAD target gene connective tissue growth factor (CTGF) (Figure 5C), whereas Ctgf mRNA expression was reduced in ABL-depleted MC3T3 cells (Figure 5D). Additionally, the ectopic expression of a mutant form of TAZ, TAZ (Ser89A), which was constitutively localized in the nucleus (27), restored the levels of Ctgf transcripts (Figure 5D) and rescued the proliferation defect observed in ABL-depleted MC3T3 cells (Figure 5E). These data demonstrate that ABL controls osteoblast proliferation through induction of the nuclear localization of TAZ and its assembly with the TEAD transcription factor.

The partitioning of cytoplasmic and nuclear TAZ is controlled by the creation of a 14-3-3-binding site following the phosphorylation of Ser89 by LATS (28). The protein phosphatase PP1 $\alpha$ dephosphorylates TAZ (Ser89), resulting in TAZ nuclear translocation (29). We asked whether active ABL affects the phosphorylation status of the LATS regulatory site on TAZ and observed that ABL (PP), but not ABL (KD), impaired TAZ (Ser89) phosphorylation and suppressed its interaction with 14-3-3 (Figure 5F). Active ABL had no effect on the interaction between TAZ and LATS1 or LATS1 (Ser909) phosphorylation (Supplemental Figure 5, A and B). However, we observed that $\mathrm{ABL}(\mathrm{PP})$, but not $\mathrm{ABL}(\mathrm{KD})$, enhanced the interaction between TAZ and PP1 $\alpha$ (Figure 5G). Coexpression of PP1 $\alpha$ or ABL (PP) with TAZ impaired TAZ (Ser89) phosphorylation and the interaction with 14-3-3, whereas this effect of PP1 $\alpha$ or ABL (PP) was reversed in the presence of the protein phosphatase inhibitor okadaic acid (Supplemental Figure 5C and Figure $5 \mathrm{H})$, demonstrating that dephosphorylation of TAZ by active ABL occurs in part through the regulation of TAZ-PP1 $\alpha$ interaction.

$T A Z$ reciprocally stabilizes and activates $A B L$ through the suppression of a ubiquitin-mediated degradation pathway. During the course of these studies, we noted that coexpression of TAZ with $A B L$ resulted in increased $A B L$ protein levels, without an alteration 
A

A Growth
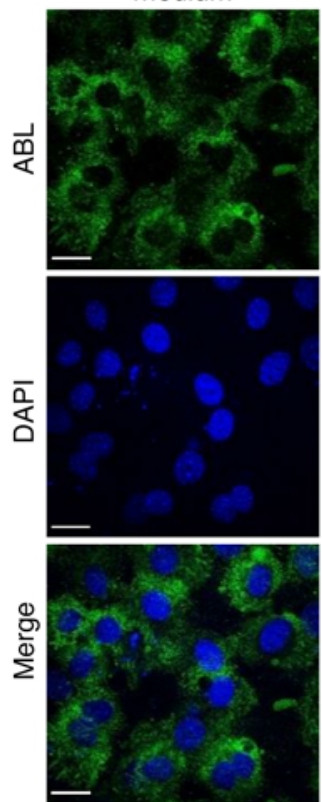

B
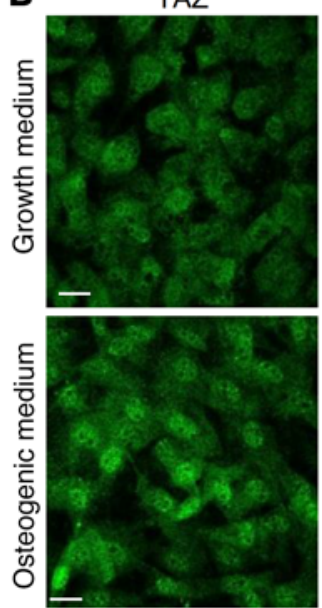

D

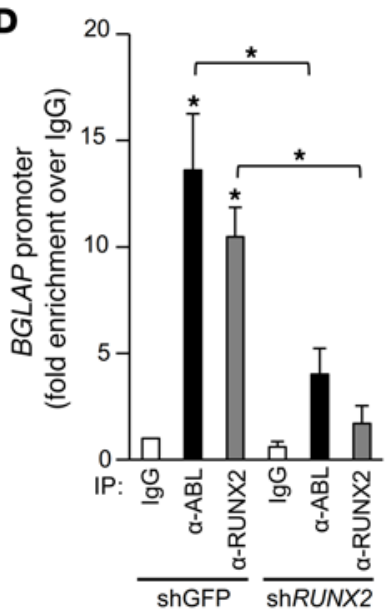

Osteogenic medium
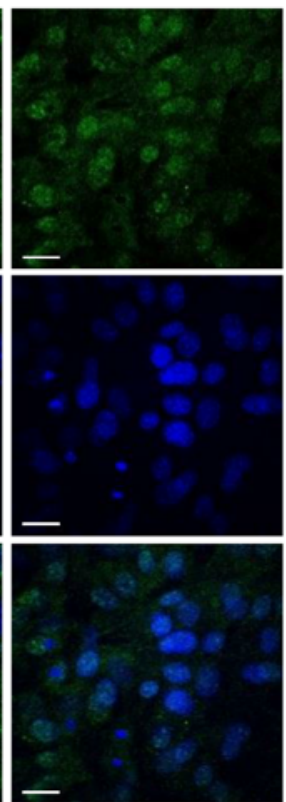

RUNX2
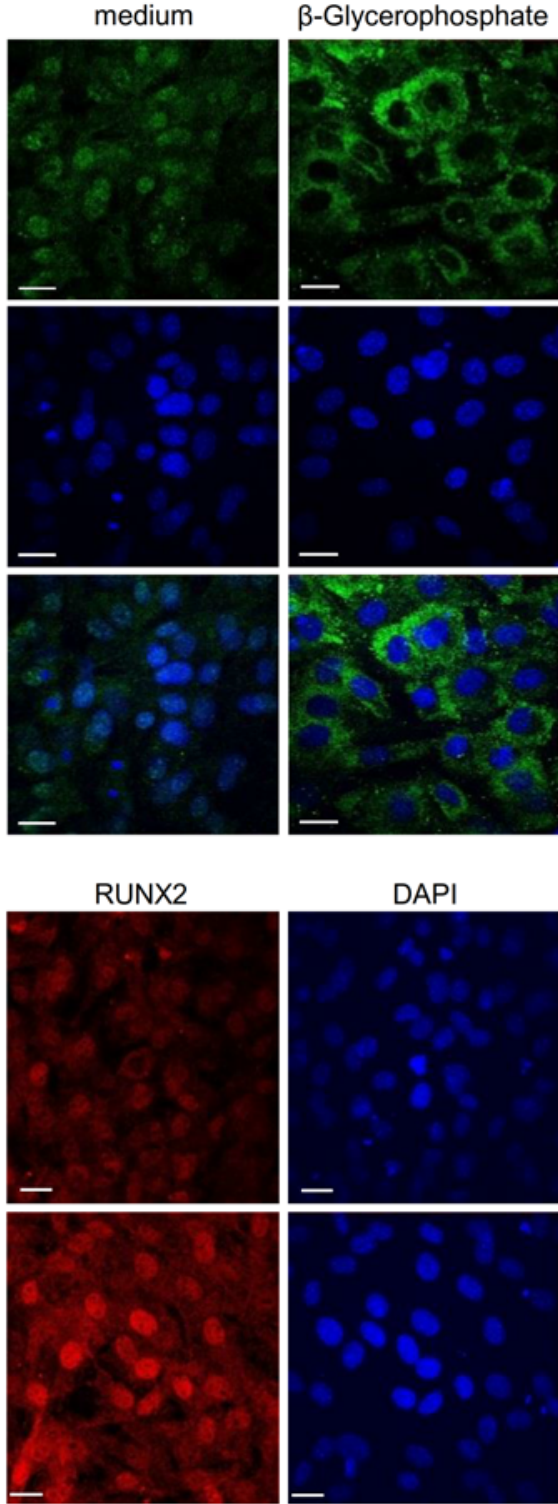

DAPI

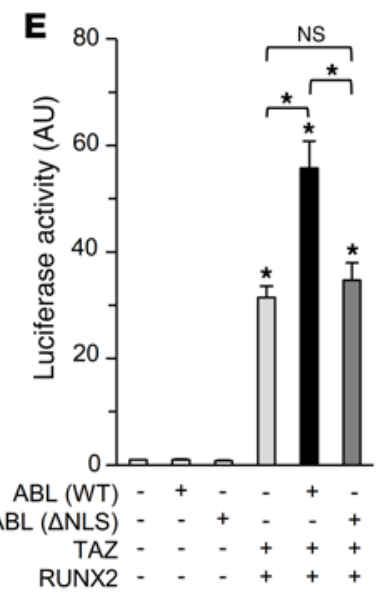

$10 \mathrm{nM}$

Dexamethasone
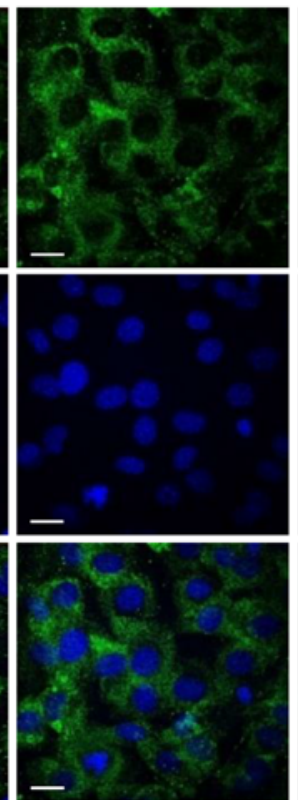

Merge

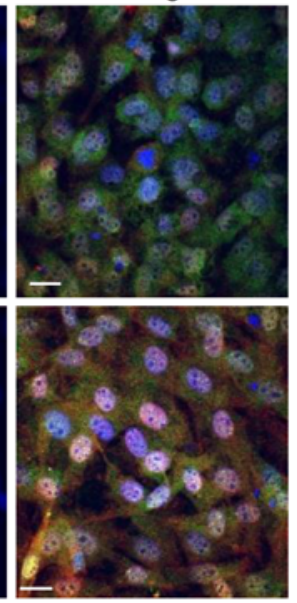

$100 \mu \mathrm{g} / \mathrm{ml}$ Ascorbic acid
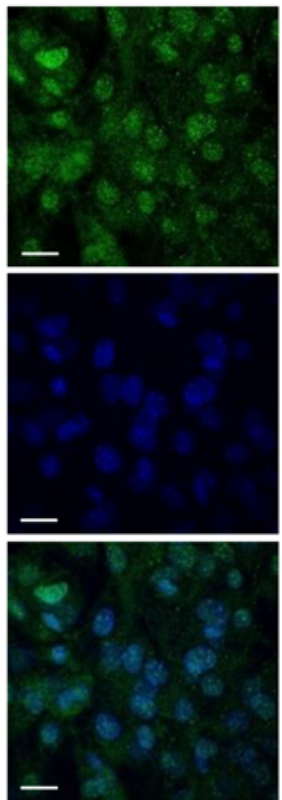

C

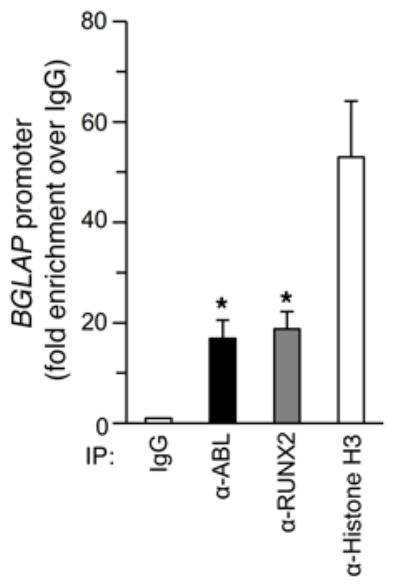

Figure 3. Nuclear ABL is required for the RUNX2-TAZ complex transcriptional activity. (A) Primary murine osteoblasts were cultured in growth medium, osteogenic medium, or growth medium supplemented with 10 $\mathrm{mM} \beta$-glycerophosphate, $10 \mathrm{nM}$ dexamethasone, or $100 \mu \mathrm{g} / \mathrm{ml}$ ascorbic acid and stained by immunofluorescence. Scale bars: $20 \mu \mathrm{m}$. The images of intracellular ABL (green) and nuclei (blue) are representative of 3 independent experiments. (B) Primary murine osteoblasts were cultured in growth medium or osteogenic medium and stained by immunofluorescence. Scale bars: $20 \mu \mathrm{m}$. The images of intracellular TAZ (green), RUNX2 (red), and nuclei (blue) are representative of 3 independent experiments. (C and $\mathbf{D}$ ) qPCR of chromatin immunoprecipitates from Saos-2 cells (C) or Saos-2 cells infected with shGFP or shRUNX2 (D). Amplicons were designed to flank the RUNX2-binding site within the BCLAP promoter. Fold enrichment represents the signal obtained after IP with a nonspecific IgG antibody. $n=3$. (E) Luciferase activity from a BCLAP reporter assay in HEK293T cells cotransfected with the indicated constructs. $n=3$. ${ }^{*} P<0.05$, by ANOVA with a Tukey-Kramer post-hoc test. Data represent the mean \pm SEM. 
A

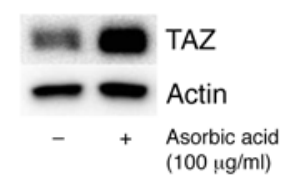

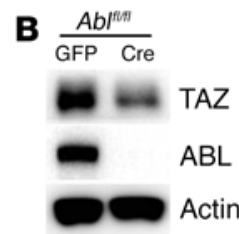

TAZ

Actin
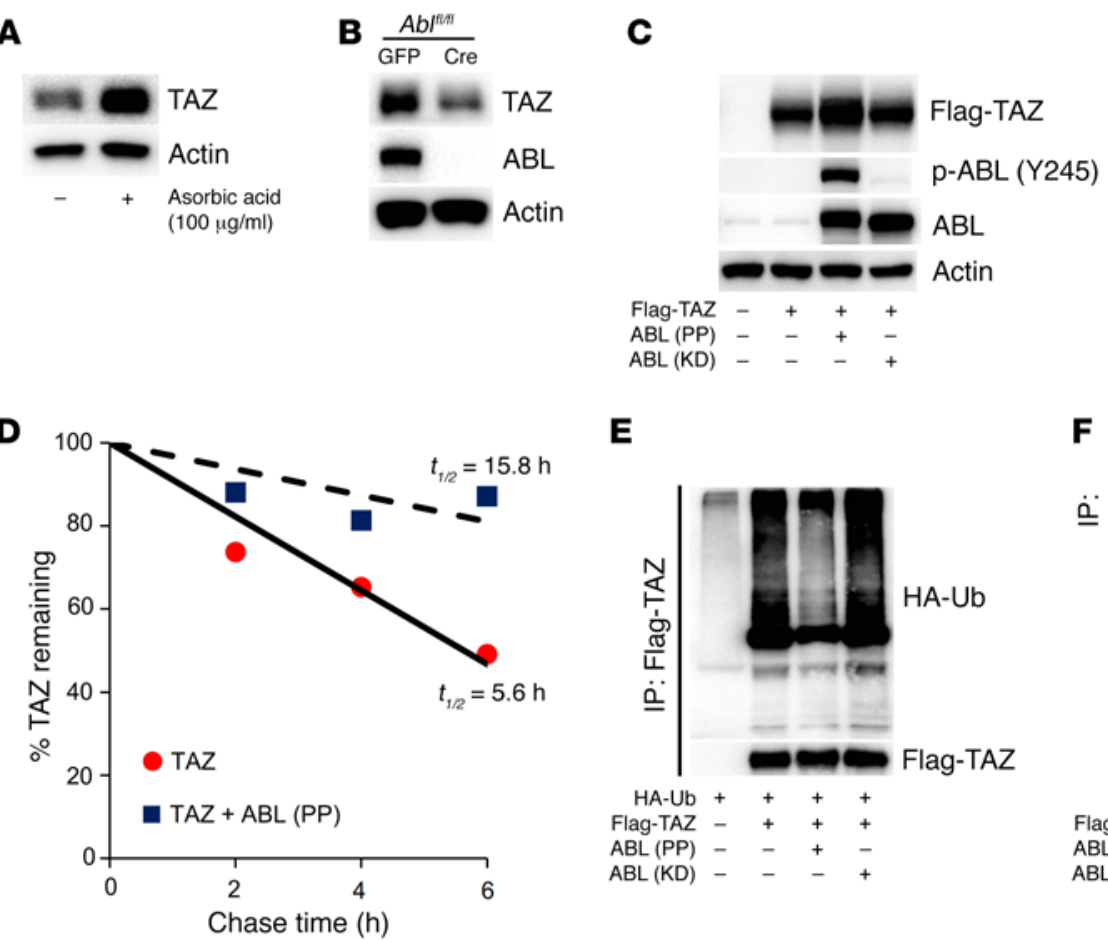

E

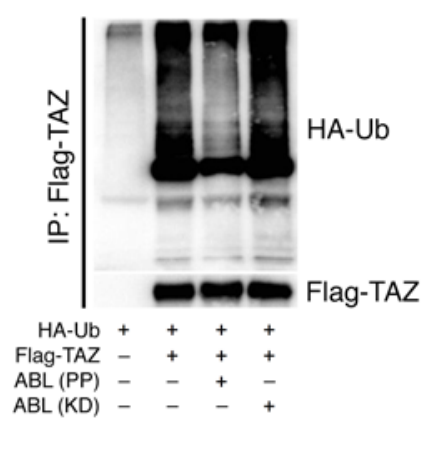

$\mathbf{F}$

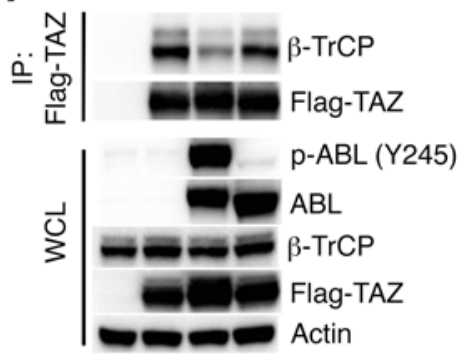

Flag-TAZ - + + +

$\mathrm{ABL}$ (PP) - - +

Figure 4. ABL stabilizes TAZ through the suppression of a ubiquitin-mediated degradation pathway. (A) Primary murine osteoblasts were cultured in growth medium or growth medium supplemented with $100 \mu \mathrm{g} / \mathrm{ml}$ ascorbic acid. WCLs were probed with the indicated antibodies for Western blot analysis. (B) Ab/flfl mouse-derived calvarial osteoblasts were infected with GFP- or Cre-expressing adenovirus to excise endogenous ABL protein (56) and cultured in osteogenic medium. WCLs were probed with the indicated antibodies for Western blot analysis. (C) HEK293T cells were cotransfected with Flag-TAZ, with or without ABL (PP or KD). WCLs were probed with the indicated antibodies for Western blot analysis. (D) HEK293T cells were cotransfected with TAZ, with or without ABL (PP). ${ }^{35}$ S-labeled TAZ protein was immunoprecipitated, separated by SDS-PACE, and analyzed by autoradiography. The percentages of TAZ protein levels are plotted as a function of time. (E) HEK293T cells cotransfected with the indicated constructs were treated with 10 $\mu \mathrm{M}$ MC132 for 4 hours prior to collection of cell lysates. Flag-TAZ immune complexes were probed with an anti-HA or anti-Flag antibody. (F) HEK293T cells were cotransfected with Flag-TAZ, with or without ABL (PP or KD). Flag-TAZ immune complexes were probed with an anti- $\beta$-TrCP antibody.

of ABL transcripts (Figure 6A and Supplemental Figure 6A), suggesting that the TAZ-mediated increase in ABL protein expression was probably posttranscriptional (30). To examine the requirement of TAZ for stabilization of endogenous $\mathrm{ABL}$, we isolated

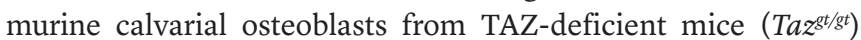
(31) and observed that ABL protein levels were decreased in the absence of TAZ (Figure 6B). In addition, we observed defective mineralization in TAZ-deficient osteoblasts (Figure 6C). ABL protein expression was also reduced in TAZ-depleted Saos- 2 cells (Supplemental Figure 6B). We observed that TAZ expression increased the half-life of ABL from 21 hours to 58 hours through the suppression of ABL ubiquitylation (Figure 6, D and E). TAZ coexpression with $\mathrm{ABL}$ increased $\mathrm{ABL}$ activity to a level similar to that observed with $\mathrm{ABL}(\mathrm{PP})$, as judged by tyrosine phosphorylation of the ABL substrate paxillin (Figure 6F). These results indicate that TAZ enhances the ABL protein stabilization and kinase activity required for osteoblast differentiation.

To define the mechanism by which ABL protein levels were increased by TAZ, we expressed 3 truncated TAZ mutants (TAZ ${ }^{2-105}$, $\mathrm{TAZ}^{106-400}$, and TAZ ${ }^{165-400}$ ) and observed that the TAZ WW domain was the common feature shared by all the mutants capable of stabilizing ABL (Figure 6G). A mutant form of TAZ lacking its WW domain, TAZ $(\triangle \mathrm{WW})$, was unable to effectively bind to or stabilize ABL (Figure 6, $\mathrm{H}$ and I).
$T A Z$ regulates $A B L$ protein stability by competitive displacement of the ABL E3-ubiquitin ligase SMURF1. We conjectured that the WW domain of TAZ might stabilize ABL through the competitive displacement of a WW domain containing E3-ubiquitin ligase. We tested 5 members of the WW domain containing HECT E3-ubiquitin ligases - SMURF1, SMURF2, NEDD4-1, NEDD4-2, and ITCH - for their ability to destabilize ABL and found that only expression of SMURF1 led to decreased ABL stability (Figure 7A). Likewise, overexpression of SMURF1 also destabilized ABL in Saos-2 cells (Supplemental Figure 7A). SMURF1 had no effect on the stabilization of a related nonreceptor tyrosine kinase, SRC (Supplemental Figure 7B), demonstrating specificity of SMURF1 for ABL. A catalytically inactive mutant form of SMURF1 (SMURF1 ${ }^{\mathrm{C} 10 \mathrm{~A}}$ ) had no effect on ABL stability (Supplemental Figure 7C), and SMURF1-mediated ABL destabilization was antagonized by proteasomal inhibition with lactacystin (Supplemental Figure 7D), suggesting that ABL stability was mediated through ubiquitin modification. Consistent with these data, SMURF1, but not the SMURF1 ${ }^{\mathrm{C} 710 \mathrm{~A}}$ mutant, catalyzed K48-linked ubiquitin modification of ABL (Figure 7B and Supplemental Figure 7E).

To test the hypothesis that TAZ alleviates the SMURF1-mediated repression of ABL through a competitive displacement mechanism, we first examined the effect of TAZ expression on SMURF1 binding to and ubiquitylation of ABL. As shown in Figure 7C, the SMURF1 ${ }^{\mathrm{C} 710 \mathrm{~A}}$ mutant efficiently bound to ABL. Coexpression of 
A
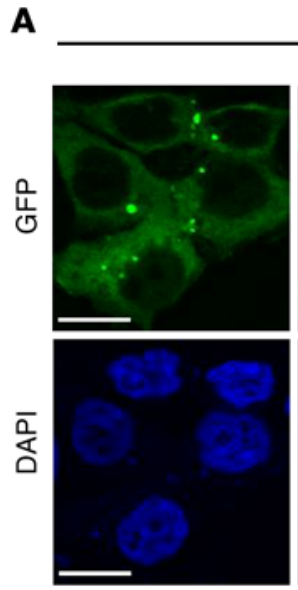

GFP-TAZ (WT) $+A B L(P P)$
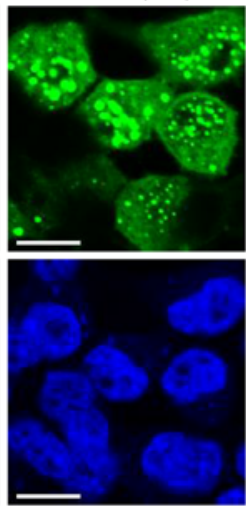

\section{$+\mathrm{ABL}(\mathrm{KD})$}
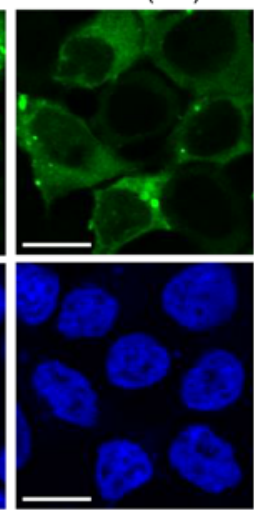

GFP-TAZ (S89A)

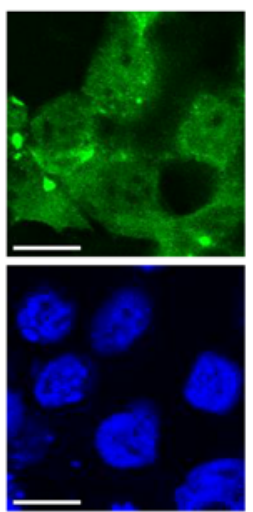

B

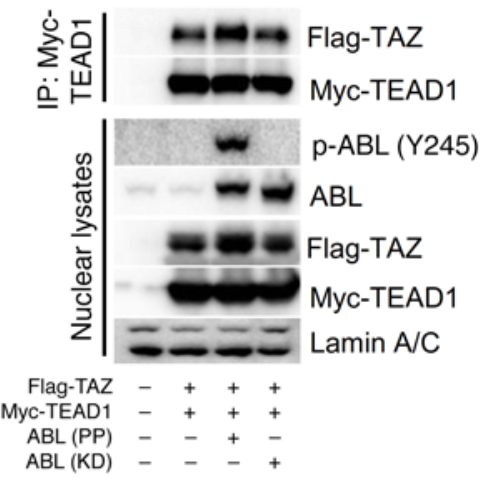

C

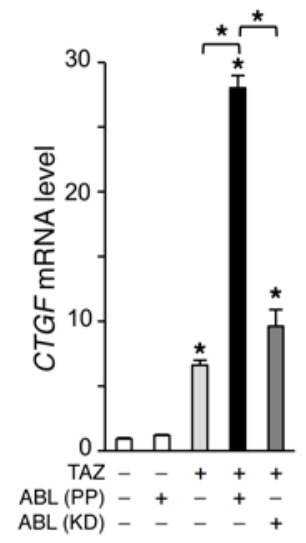

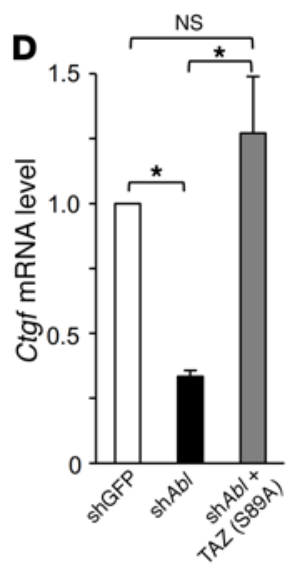

E

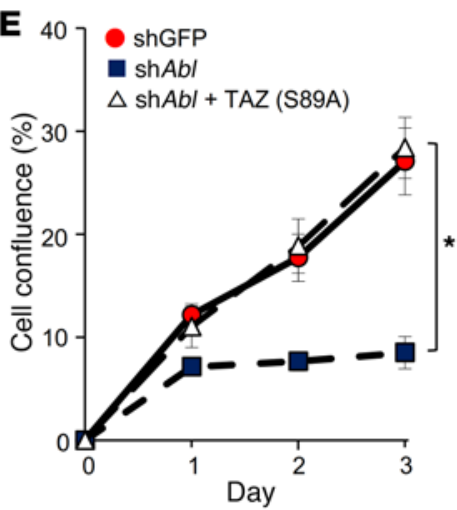

$\mathbf{F}$

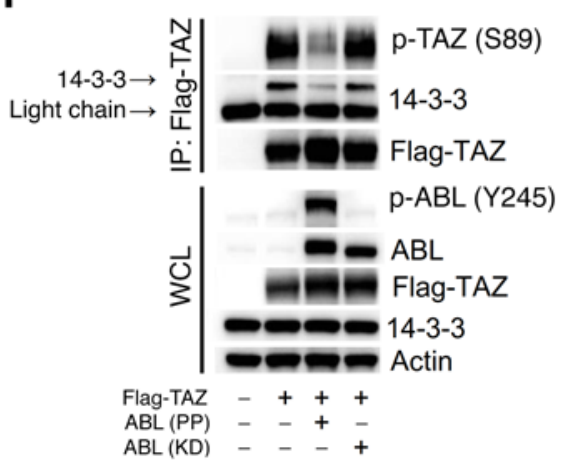

G

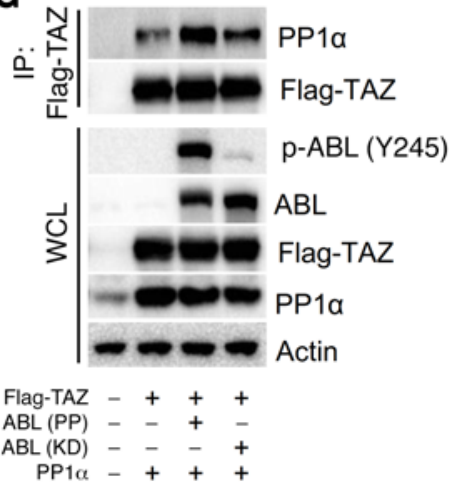

H

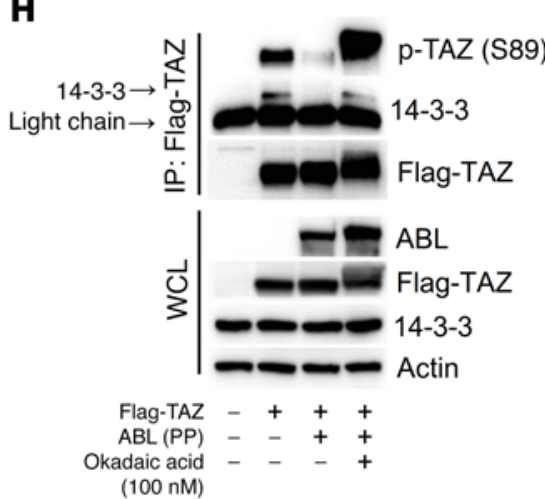

Figure 5. ABL stabilizes the TAZ-TEAD complex required for osteoblast expansion. (A) HEK293T cells were cotransfected with GFP-TAZ (WT or S89A), with or without $A B L(P P$ or KD), and stained by immunofluorescence. Scale bars: $10 \mu \mathrm{m}$. The images of intracellular TAZ (green) and nuclei (blue) are representative of 3 independent experiments. (B) HEK293T cells were cotransfected with Flag-TAZ and Myc-TEAD1, with or without ABL (PP or KD). The nuclear compartment was extracted from the cells, and Myc-TEAD1 immune complexes were probed with an anti-Flag antibody. (C) qPCR analysis of Ctgf mRNA expression in HEK293T cells cotransfected with TAZ, with or without ABL (PP or KD). $n=3$. (D) qPCR analysis of Ctgf mRNA expression in MC3T3 cells infected with shGFP or shAbL, with or without a retroviral vector expressing TAZ (S89A). $n=3$. (E) Growth curves of MC3T3 cells infected with shGFP or shABL, with or without a retroviral expressing TAZ (S89A), and cultured for 3 days. $n=3$. (F) HEK293T cells were cotransfected with Flag-TAZ, with or without ABL (PP or KD). Flag-TAZ immune complexes were probed with an anti-p-YAP (Ser127) or anti-14-3-3 antibody. (C) HEK293T cells were cotransfected with Flag-TAZ and PP1 $\alpha$, with or without ABL (PP or KD). Flag-TAZ immune complexes were probed with an anti-PP1 $\alpha$ antibody. (H) HEK293T cells cotransfected with Flag-TAZ, with or without $A B L(P P)$, were cultured for 4 hours in the presence or absence of okadaic acid (100 nM). Flag-TAZ immune complexes were probed with an anti-p-YAP (Ser127) or anti-14-3-3 antibody. ${ }^{*} P<0.05$, by ANOVA with a Tukey-Kramer post-hoc test. Data represent the mean \pm SEM.

TAZ potently inhibited the interaction of SMURF1 with ABL in a dose-dependent manner (Figure 7C) and diminished SMURF1mediated ABL ubiquitylation (Figure 7D). Moreover, the TAZ $(\triangle \mathrm{WW})$ mutant was unable to displace SMURF1 from ABL (Figure
7E). These data are consistent with a model in which TAZ stabilizes ABL through the competitive displacement of SMURF1.

Last, to test whether endogenous $\mathrm{ABL}$ is a physiologic substrate of SMURF1 in osteoblasts, we measured ABL levels in cal- 
A

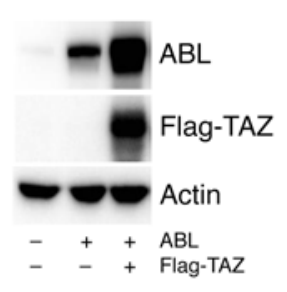

B

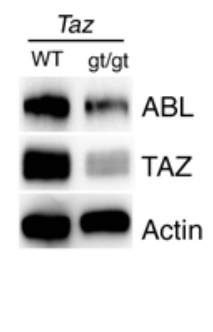

C

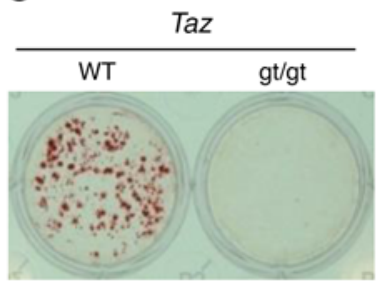

D

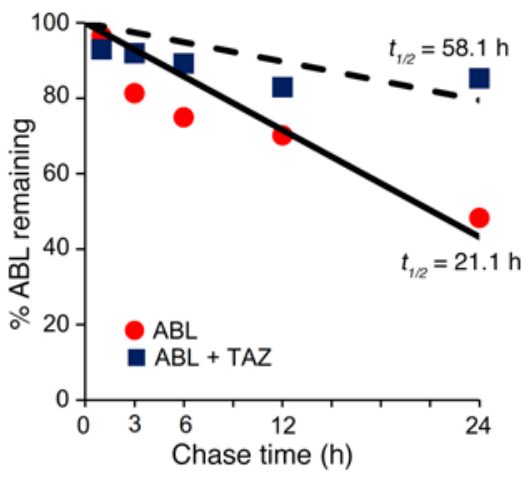

E

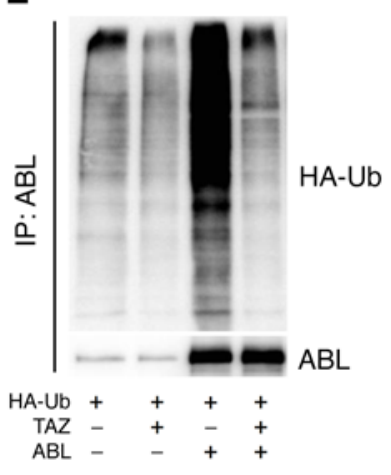

$\mathbf{F}$
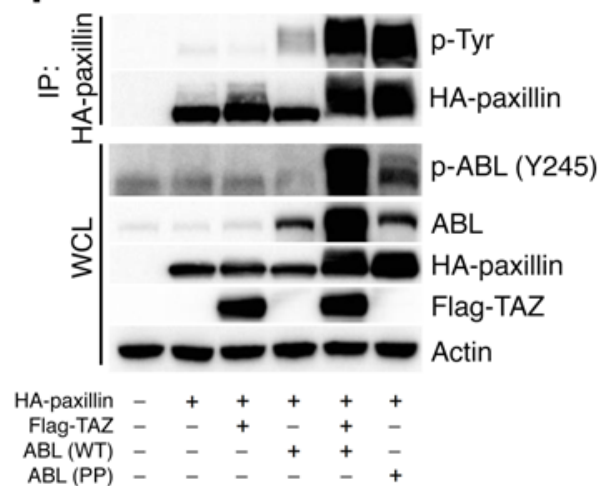

I
H

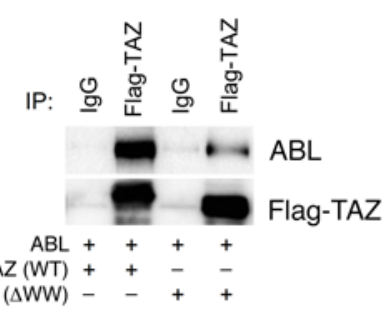

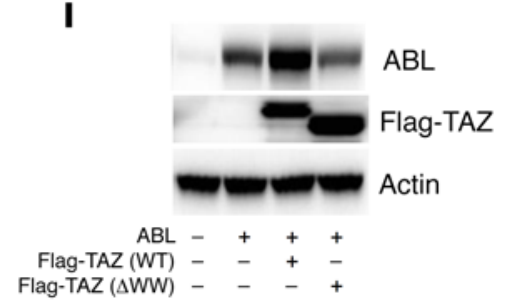

G

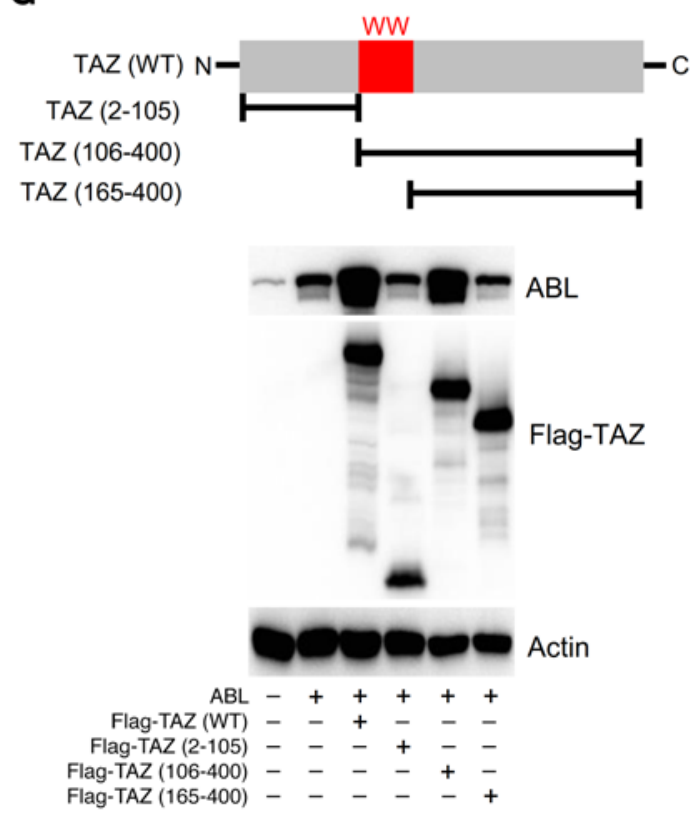

Figure 6. TAZ reciprocally stabilizes and activates ABL through the suppression of a ubiquitin-mediated degradation pathway. (A) HEK293T cells were cotransfected with ABL, with or without Flag-TAZ. WCLs were probed with the indicated antibodies for Western blot analysis. (B) Primary murine osteoblasts from WT or Taz ${ }^{g t / g t}$ (gt/gt) mice were cultured in osteogenic medium. WCLs were probed with the indicated antibodies for Western blot analysis. (C) Osteoblasts in B were cultured in osteogenic medium and stained with alizarin red S solution. $n=3$. (D) HEK293T cells were cotransfected with ABL, with or without TAZ. ${ }^{35}$ S-labeled ABL protein was immunoprecipitated, separated by SDS-PACE, and analyzed by autoradiography. The percentages of ABL protein levels are plotted as a function of time. (E) HEK293T cells cotransfected with the indicated constructs were treated with $10 \mu \mathrm{M}$ MG132 for 4 hours prior to collection of cell lysates. ABL immune complexes were probed with an anti-HA or anti-ABL antibody. (F) HEK293T cells were cotransfected with HA-paxillin and Flag-TAZ, with or without ABL (WT or PP). HA-paxillin immune complexes were probed with an anti-p-Tyr antibody. (C) HEK293T cells were cotransfected with $A B L$ and the indicated Flag-TAZ truncation mutant. WCLs were probed with the indicated antibodies for Western blot analysis. N, N-terminal; C, C-terminal. (H and I) HEK293T cells were cotransfected with ABL and either full-length TAZ [Flag-TAZ (WT)] or TAZ lacking the WW domain [Flag-TAZ $(\triangle \mathrm{WW})$ ]. Flag-TAZ immune complexes $(\mathbf{H})$ or WCLs (I) were probed with the indicated antibodies.

varial osteoblasts derived from Smurf1-KO mice and observed that ABL protein levels were elevated 2-fold in the absence of SMURF1 (Figure 7F). While loss of SMURF1 enhanced in vitro osteoblast mineralization as previously described (Figure 7G) (32), we showed that depletion of ABL abolished this effect (Figure 7, F and G). These genetic and biochemical data identify a bona fide ABL E3-ubiquitin ligase and show that TAZ antagonizes SMURF1 repression of ABL protein levels.

The ABL-TAZ amplification loop required for osteoblastogenesis is regulated by the adapter protein $3 B P 2$. We next investigated the upstream signaling pathway that is required for initiation of the ABL-TAZ amplification loop during osteoblastogenesis.
Our previous demonstration that endogenous levels of active ABL were reduced in 3BP2-deficient osteoblasts (19) suggested a functional link between 3BP2 and ABL-TAZ stabilization. We therefore analyzed the protein expression levels of $\mathrm{ABL}$ and TAZ in Sh3bp2-- calvarial osteoblasts and found that both nuclear ABL and TAZ protein levels were reduced with defective osteoblast mineralization (Figure 8A and Supplemental Figure 8). We hypothesized that TAZ levels were regulated downstream of 3BP2-mediated activation of ABL and queried whether the reduced nuclear TAZ levels observed in Sh3bp $2^{---}$calvarial osteoblasts could be rescued by the ectopic expression of FKBP-ABL. We observed that the expression of FKBP-ABL restored endoge- 

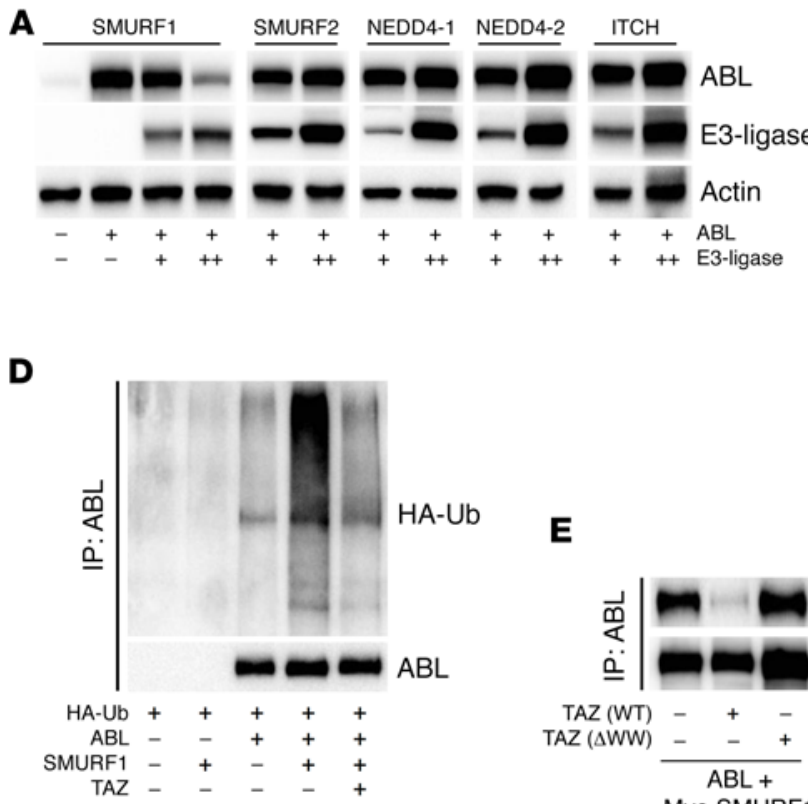

E

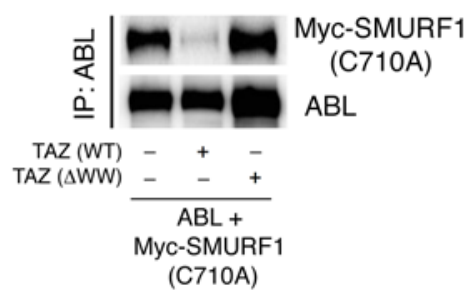

B

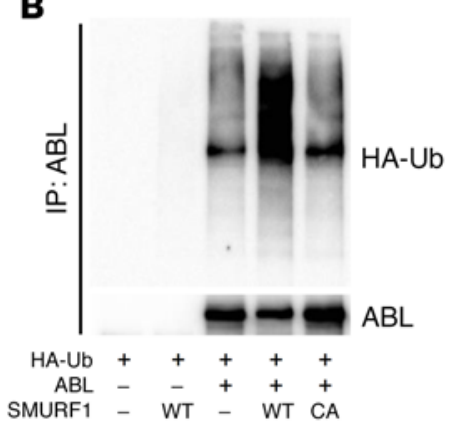

C

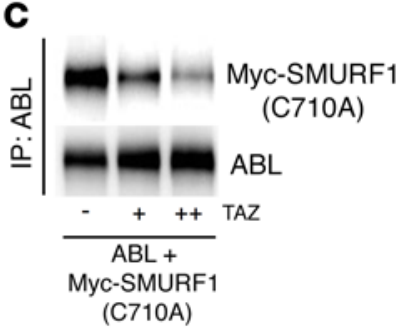

$\mathbf{F}$

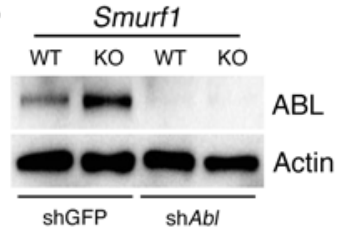

G

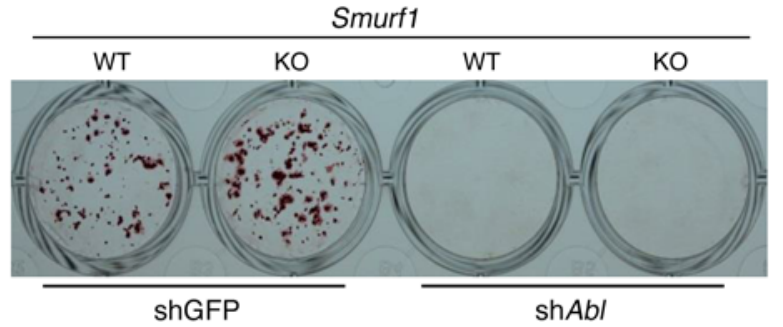

Figure 7. TAZ regulates ABL protein stability by competitive displacement of the ABL E3-ubiquitin ligase SMURF1. (A) HEK 293T cells were cotransfected with $A B L$, with or without the indicated HECT domain-containing E3-ligases. The specific E3-ligase transfected into cells is indicated at the top panel of Western blots (SMURF1, SMURF2, NEDD4-1, NEDD4-2, and ITCH; 0-0.5 $\mu \mathrm{g} /$ well). WCLs were probed with the indicated antibodies for Western blot analysis. (B) HEK293T cells cotransfected with the indicated constructs, with or without SMURF1 (WT or C710A mutant [CA]), were treated with $10 \mu M$ MG132 for 4 hours prior to collection of cell lysates. ABL immune complexes were probed with an anti-HA or anti-ABL antibody. (C) HEK293T cells were cotransfected with ABL, Myc-SMURF1 (C710A), and increasing amounts of the TAZ construct. ABL immune complexes were probed with an anti-Myc antibody. (D) HEK293T cells cotransfected with the indicated constructs were treated with $10 \mu \mathrm{M} \mathrm{MC132}$ for 4 hours prior to collection of cell lysates. ABL immune complexes were probed with an anti-HA or anti-ABL antibody. (E) HEK293T cells were cotransfected with ABL and Myc-SMURF1 (C710A), with or without TAZ (WT or $\triangle \mathrm{WW}$ ). ABL immune complexes were probed with an anti-Myc antibody. (F) Primary murine osteoblasts from WT or Smurf1 $1^{-/-}$(KO) mice were infected with shGFP or ShABL and cultured in osteogenic medium. WCLs were probed with the indicated antibodies for Western blot analysis. (C) Osteoblasts in $\mathbf{F}$ were cultured in osteogenic medium and stained with alizarin red $\mathrm{S}$ solution. $n=3$.

nous nuclear TAZ protein in Sh3bp2-- osteoblasts to normal levels (Figure 8B) and rescued the mineralization defect observed in 3BP2-deficient osteoblasts, as we previously reported (Figure 8C) (19). These data provide evidence that 3BP2 is needed to initiate the positive amplification loop between ABL and TAZ that is necessary for osteoblastogenesis.

We show in the present study that ABL is essential for osteoblast expansion, differentiation, and mineralization through the interaction with TAZ and RUNX2, leading to embryonic bone synthesis. Last, we investigated whether ABL regulated the development of other mesenchymal lineages and observed that active FKBP-ABL (WT), but not kinase-dead FKBP-ABL (KD), dramatically suppressed adipocyte differentiation in NIH3T3 cells (Figure 8, D and E) and the transcriptional activity of PPAR (Figure 8, F and $G$ ), thus demonstrating that ABL enhances osteoblastogenesis while suppressing adipogenesis.
These data show that ABL activation initiated by 3BP2 regulates the ABL and TAZ protein levels required for osteoblastogenesis, whereas active $A B L$ antagonizes adipocyte differentiation through the suppression of PPAR $\gamma$ activity.

\section{Discussion}

Protein stabilization of $A B L$ and $T A Z$ is required for normal osteoblastogenesis during embryonic skeletal development. In distinction to autoregulatory transcription networks that control the development of cellular identity, we have identified what we believe to be a novel regulatory strategy involving the reciprocal stabilization of $\mathrm{ABL}$ and TAZ protein required for osteoblast maturation. In our model, 3BP2 activates ABL, which triggers the mutual stabilization of ABL and TAZ through the exclusion of their respective E3-ubiquitin ligases, SMURF1 and $\beta$-TrCP (Figure $8 \mathrm{H}$ ). The accumulation of $\mathrm{ABL}$ in the nucleus during osteoblastogenesis serves to assemble 
A

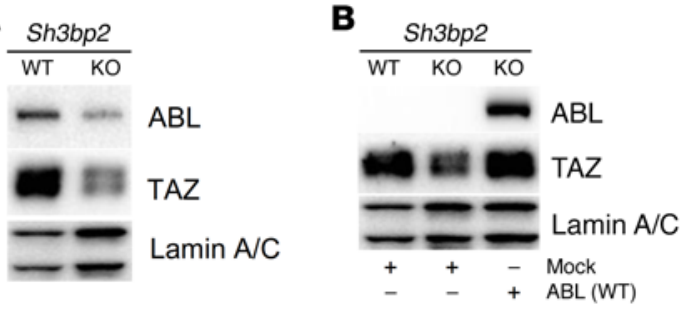

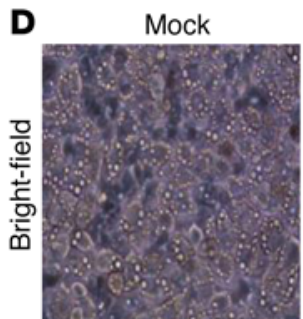

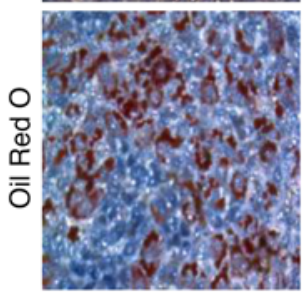

F

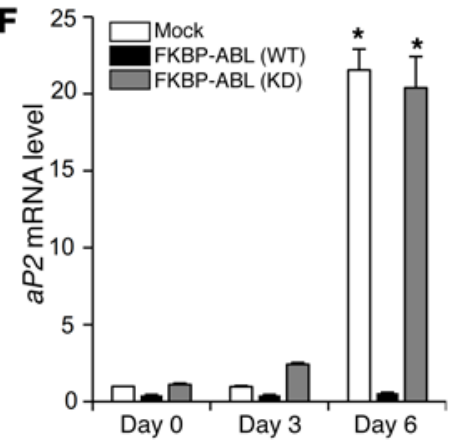

$\mathrm{ABL}$ (WT)

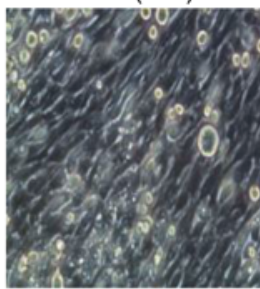

$\mathrm{ABL}(\mathrm{KD})$

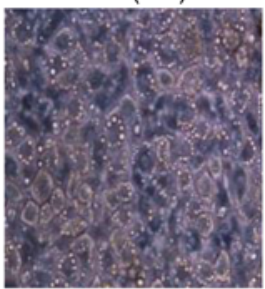

C

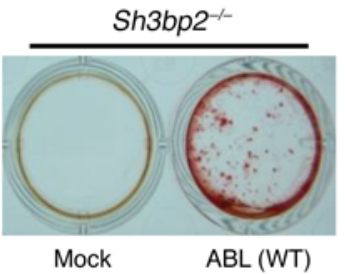

E

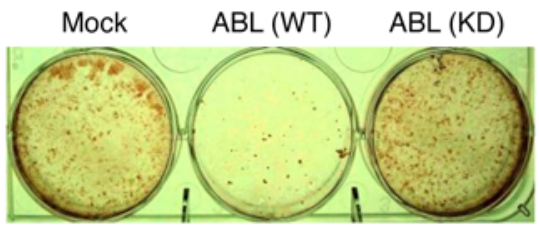

H

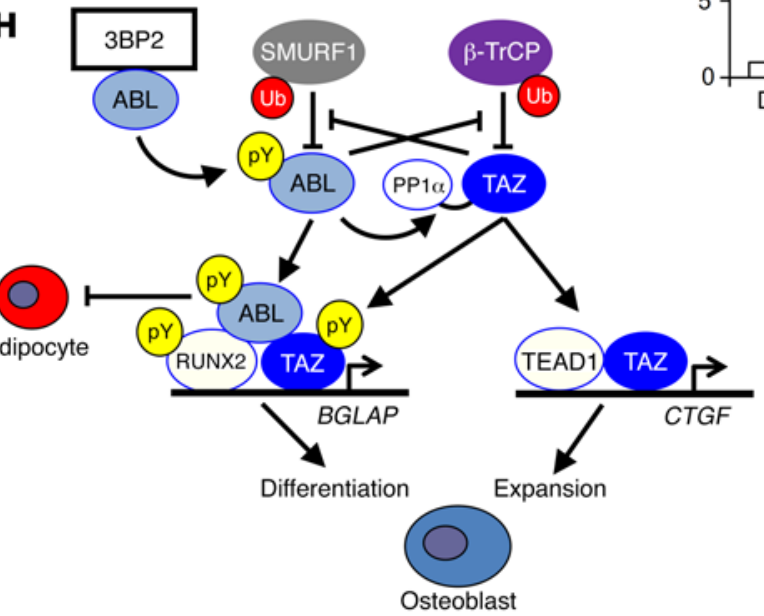

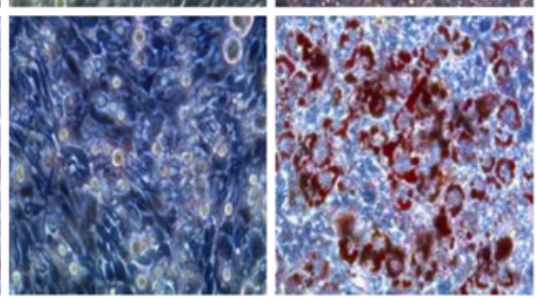

G

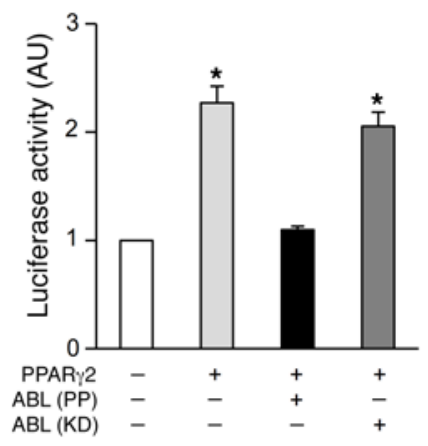

Figure 8. The ABL-TAZ amplification loop required for osteoblastogenesis is regulated by the adapter protein 3BP2. (A) Primary murine osteoblasts from WT or Sh3bp2-- (KO) mice were cultured in osteogenic medium. Nuclear lysates were probed with the indicated antibodies for Western blot analysis. (B) Primary murine osteoblasts from WT or Sh3bp2 $2^{-1-}$ (KO) mice were infected with an empty vector control or FKBP-ABL-expressing retroviral vector (WT) and cultured in osteogenic medium. Nuclear lysates were probed with the indicated antibodies for Western blot analysis. (C) Osteoblasts in B were cultured in osteogenic medium and stained with alizarin red S solution. $n=3$. (D) NIH3T3 cells infected with an empty vector control or a retroviral vector expressing FKBP-ABL (WT or KD) were cultured in adipogenic medium and stained with oil red 0 solution. Upper panel: bright-field images; bottom panel: oil red $0-$ stained images; original magnification $\times 200 . n=3$. (E) Culture plate images of the oil red 0 staining in $\mathbf{D} . n=3$. (F) qPCR analysis of $a P 2$ mRNA expression in cells in $\mathbf{D}$ and $\mathbf{E}$ cultured for 3 to 6 days. $n=3$. Data are presented as the mean \pm SEM. (G) Luciferase activity from an $a P 2$ reporter assay in HEK293T cells cotransfected with the indicated constructs. $n=3 .{ }^{*} P<0.05$, by ANOVA with a Tukey-Kramer post-hoc test. (H) Schematic model of reciprocal protein stabilization of $A B L$ and $T A Z$, which regulates osteoblastogenesis through the activation of RUNX2.

and activate the RUNX2-TAZ master transcription factor complex that drives osteoblast differentiation and mineralization through tyrosine phosphorylation of these proteins (Figure $8 \mathrm{H}$ ).

We propose that active $\mathrm{ABL}$ is a regulator of the mesenchymal maturation program, depending on its target substrate. During embryonic bone synthesis, ABL reinforces the active RUNX2-TAZ complex that is required for osteoblastogenesis. Our findings support those of recent studies in rats treated with the ABL inhibitor dasatinib, in which delayed fetal ossification was reported $(33,34)$. In distinction to its role within the osteoblast lineage, ABL suppresses myogenic differentiation by downregulating the expression and activity of $\mathrm{MyoD}(35,36)$. Additionally, we found that active ABL antagonized adipocyte differentiation (Figure 8H). Since TAZ suppresses PPAR $\gamma$ transcriptional activity and adipogenesis (10), it is conceivable that ABL-mediated TAZ stabilization could similarly control the adipocyte maturation program. Further investigation 
will be necessary to clarify the molecular mechanisms by which active ABL regulates PPAR $\gamma$ activity and adipogenesis.

$A B L$ regulates osteoblast expansion through the TAZ-TEAD complex. The Hippo pathway transcriptional coactivators YAP and TAZ are both phosphorylated by ABL under a variety of distinct physiologic states. TAZ is phosphorylated by ABL in response to hyperosmotic stress in renal tubule cells (37). ABL, TAZ, and the nuclear factor of activated T cells 5 (NFAT5) form a complex that regulates the expression of betaine-GABA transporter 1 (BGT1) and sodium/myo-inositol cotransporter (SMIT), both of which are required for adaptation to osmotic stress. YAP is phosphorylated by $\mathrm{ABL}$ to induce apoptosis following DNA damage (25). Our study unveils a new interaction between ABL and components of the Hippo pathway. We show that ABL stabilized TAZ and enhanced the interaction between TAZ and the PP1 $\alpha$ phosphatase, leading to dephosphorylation of the 14-3-3-binding site. ABL triggers translocation of TAZ to the nucleus, where it can form a complex with TEAD to drive proliferation (8). Depletion of ABL in osteoblasts reduced CTGF expression and cell growth, which was rescued by the expression of a nuclear mutant form of TAZ. ABL has previously been reported to regulate osteoblast expansion through the BMP receptor (38). In $\mathrm{Abl}^{-/-}$mesenchymal osteoprogenitor cells, the noncanonical BMP/ERK pathway is activated, leading to $\mathrm{p} 16^{\mathrm{INK} 4 \mathrm{a}}$ upregulation and cell senescence (38). Our data provide insight into the role of ABL for osteoblast proliferation and expand the concept that $\mathrm{ABL}$ is an important kinase that regulates TAZ and YAP in multiple lineages.

SMURF1 is an ABL E3-ubiquitin ligase. We have identified SMURF1 as an ABL E3-ubiquitin ligase in osteoblasts that is competitively displaced by TAZ to attenuate ABL ubiquitylation and degradation. SMURF1 is a known negative regulator of osteoblastogenesis, as Smurf1 ${ }^{-/-}$mice exhibit increased bone mass with enhanced osteoblast differentiation (32). We showed that ABL is a physiologic substrate of SMURF1 in primary osteoblasts and demonstrated that the enhanced in vitro mineralization observed in SMURF1-deficient osteoblasts is in part dependent on ABL. SMURF1 has other substrates involved in osteoblast development in addition to ABL, including RUNX2, SMAD1, and MEKK2 (32, 39-44), indicating that SMURF1 controls a critical nexus of signaling proteins that regulate bone synthesis.

We report a mechanistic strategy, whereby the interplay between ABL and TAZ creates an amplification loop that stabilizes and activates the RUNX2-TAZ transcriptional complex needed to "lock in" mesenchymal development toward the osteoblast lineage.

\section{Methods}

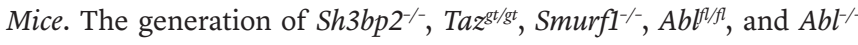
mice has been described previously (31, 45-49).

Cell cultures. All cultures were maintained in a $5 \% \mathrm{CO}_{2}$ environment at $37^{\circ} \mathrm{C}$. HEK $293 \mathrm{~T}$ cells (ATCC) were cultured in DMEM (Wisent) supplemented with 10\% FBS (Wisent). NIH3T3 cells (ATCC) were cultured in DMEM supplemented with $10 \%$ bovine calf serum (Wisent). Saos-2 cells (ATCC) were cultured in McCoy's 5A Modified Medium (Wisent) supplemented with 15\% FBS. MC3T3 cells (ATCC) were cultured in $\alpha$-MEM (Wisent) supplemented with $10 \%$ FBS.

Osteoblast cultures. Neonatal calvarium-derived osteoblasts were harvested and cultured as described previously (50). Osteoblast dif- ferentiation was induced by culturing cells in osteogenic medium ( $\alpha$-MEM containing 10\% FBS, $100 \mu \mathrm{g} / \mathrm{ml}$ ascorbic acid, $10 \mathrm{mM}$ $\beta$-glycerophosphate, and $10 \mathrm{mM}$ dexamethasone) for 21 days. Alizarin red staining of mineralization was accomplished by cell fixation in $4 \%$ formaldehyde for 30 minutes, followed by staining with $0.1 \%$ alizarin red S solution ( $\mathrm{pH} 4.8$ ) for 20 minutes.

Adipocyte differentiation. Adipocyte differentiation was induced by culturing cells in adipogenic medium (DMEM containing $10 \% \mathrm{FBS}$, $10^{-6} \mathrm{M}$ dexamethasone, $0.5 \mathrm{mM}$ 3-isobutyl-1-methylxanthine, $10 \mu \mathrm{g} /$ $\mathrm{ml}$ insulin, and $10 \mu \mathrm{M}$ troglitazone). Adipocytes were evaluated by oil red $\mathrm{O}$ staining, which was performed by cell fixation in $4 \%$ formaldehyde for 15 minutes, followed by staining with filtered $0.6 \%$ oil red $\mathrm{O}$ solution in $60 \%$ isopropanol. Oil red O-positive cells were determined by cytoplasmic fat globule cells.

Microcomputed tomography. The samples were imaged using a small animal Inveon CT Scanner (Siemens Preclinical Solutions). Both skulls and femurs were imaged with the following parameters: voltage $80 \mathrm{kV}$; current $500 \mu \mathrm{A}$; exposure time 1,600 ms; no filter; final resolution of $18.40 \mu \mathrm{m}$. The reconstructed data sets were analyzed with Inveon Research Workplace Software, version 4.0. For histomorphometric analysis, regions of interest were designed throughout the full width and thickness of the right femurs, including both cortical and trabecular structures. A 3D rendering of the imaged samples was also produced.

Histological analysis. For skeletal alizarin red and Alcian blue staining, newborn pups were fixed in $95 \%$ ethanol and transferred to acetone, followed by staining as described previously (12).

Histomorphometry. In vivo osteoblast parameters were generated from undecalcified tibial sections embedded in methyl methacrylate and stained with Goldner's trichrome. In vivo osteoclast parameters were generated from decalcified tibial sections embedded in paraffin and stained for tartrate-resistant acid phosphatase (TRAP). Images were analyzed using BIOQUANT software (BIOQUANT Image Analysis Corp.) (19).

Reagents and antibodies. Unless stated otherwise, all chemicals were purchased from Sigma-Aldrich. The following antibodies were used: 17. anti-phosphorylated Tyr (anti-p-Tyr) (for co-IP) (cat. 9411), antip-ABL (Y245) (cat. 2861), anti-SRC (cat. 2109), anti-p-YAP (Ser127) (cat. 4911), anti-lamin A/C (cat. 2032), anti- $\beta$-TrCP (cat. 4394), antiLATS1 (cat. 3477), and anti-p-LATS1 (Ser909) (cat. 9157) (Cell Signaling Technology); anti-ABL (cat. 554148) and anti-TAZ (cat. 560235) (BD Pharmingen); anti- Flag M2 (F3165) and anti-Myc (M4439) (Sigma-Aldrich); anti-actin (cat. sc-47778) and anti-14-3-3 (cat. sc-133232) (Santa Cruz Biotechnology Inc.); anti-HA (11867423001) (Roche); anti-RUNX2 (D-130-3) (MBL International); anti-p-Tyr (4G10) (cat. 05-321) and anti-V5 (AB3792) (EMD Millipore); and anti-3BP2 (H00006452-M01) (Abnova). Halt Protease and Phosphatase Inhibitor Cocktail was purchased from Thermo Fisher Scientific.

Plasmids. ABL (WT, PP, KD, or $\triangle \mathrm{NLS}$ ) and HA-Ub (WT or K48R) plasmids were from the laboratory of Giulio Superti-Furga; TAZ (WT or $\triangle \mathrm{WW}$ ), SMURF1 (WT or C710A), SMURF2, NEDD4-1 or -2, ITCH, p6OSE2-luc, and phRL-CMV plasmids were from the laboratory of Jeffrey L. Wrana; RUNX2 (WT), PPAR $\gamma$ 2, and aP2-luc plasmids were gifts of Michael B. Yaffe (Koch Institute for Integrative Cancer Research, Massachusetts Institute of Technology, Cambridge, Massachusetts, USA); and the HA-paxillin plasmid was a gift of Oliver Hantschel (Swiss Institute for Experimental Cancer Research, School of Life 
Sciences, École polytechnique fédérale de Lausanne, Lausanne, Switzerland). The TEAD1 plasmid was purchased from Addgene (51), and the PP1 $\alpha$ plasmid was purchased from OriGene Technologies. The RUNX2 (YF) plasmid was synthesized by Life Technologies (Thermo Fisher Scientific). The TAZ (YF or S89A) plasmid was constructed using the Site-Directed Mutagenesis Kit (Stratagene).

RNA extraction and quantitative real-time PCR analysis. Total cellular RNA was extracted using the RNeasy Plus Mini Kit (QIAGEN). The ImProm-II Reverse Transcription System (Promega) was used for reverse transcription, and quantitative real-time PCR (qPCR) was performed using the StepOnePlus Real-Time PCR System (Applied Biosystems) according to the manufacturer's protocol. The primer sequences are provided in the Supplemental Methods. The relative expression of each mRNA was calculated by the $\triangle \mathrm{Ct}$ method.

Expression of FKBP-ABL and TAZ (S89A) retroviral vectors. FKBP$\mathrm{ABL}$ and TAZ (S89A) retroviral vectors were constructed as described previously (19). HEK293T cells were cotransfected with an empty vector control (Mock), pMx-FKBP-ABL, or pMx-TAZ (S89A) with pSV and pVSVG using a CalPhos Mammalian Transfection Kit (Clontech). Osteoblasts were infected and then cultured in osteogenic medium as described previously (19).

Lentiviral transduction. $\mathrm{pLKO} .1$ lentiviral vectors expressing shRNAs targeting RUNX2 (shRUNX2), TAZ (shTAZ), ABL (shABL), or a nonspecific GFP sequence (shGFP) were cotransfected into HEK293T cells with pPAX2 and pVSVG (Addgene) using the X-tremeGENE 9 DNA Transfection Reagent (Roche). The virus was collected 48 hours after transfection, and cells were infected as described previously (19).

ChIP. Endogenous ABL and RUNX2 were immunoprecipitated from Saos-2 cell lysates with a SimpleChiP Enzymatic Chromatin IP Kit (Cell Signaling Technology; product number 9003) according to the manufacturer's protocol. IPs were performed with anti-ABL (Santa Cruz Biotechnology Inc.; K-12, catalog sc-131), anti-RUNX2, or antihistone H3 (Cell Signaling Technology) antibody or control IgG. ChIP amplicons were designed to flank the RUNX2-binding site within the $B G L A P$ promoter as described previously (25).

Western blot analysis and co-IP. Cells were lysed with Nonidet P-40 (NP-40) buffer (20 mM Tris, pH 8.0, $137 \mathrm{mM} \mathrm{NaCl}, 1 \% \mathrm{NP}-40,2 \mathrm{mM}$ EDTA) or RIPA buffer (50 mM Tris, pH 7.5, 150 mM NaCl, 1\% NP-40, $0.1 \%$ SDS, $0.25 \%$ sodium deoxycholate, $1 \mathrm{mM}$ EDTA) supplemented with protease and phosphatase inhibitors. Lysates were cleared by centrifugation for 10 minutes at $18,000 \mathrm{~g}$ and $4^{\circ} \mathrm{C}$. IP was performed at $4^{\circ} \mathrm{C}$ with the indicated antibodies, and the products were collected on Dynabeads Protein A or G (Life Technologies, Thermo Fisher Scientific) (Figure 1F; Figure 2 A, B, D-I; Figure 4F; Figure 5, B, F-H; Figure 6, F and H; Figure 7, C and E; Supplemental Figure 2, A, B, D, F; Supplemental Figure 5, A-C). These methods and antibodies were used for all the IP experiments. For Western blotting, protein in whole-cell lysates (WCLs) were resolved by SDS-PAGE and transferred to an Immobilon PVDF membrane (EMD Millipore). Membranes were blocked in 5\% BSA or $5 \%$ nonfat dried milk in PBS plus $0.1 \%$ Tween-20 (PBST). To analyze the interaction in the nuclear compartment, we used a Nuclear Complex Co-IP Kit (Active Motif) according to the manufacturer's protocol. p-TAZ (Ser89) was detected by an anti-p-YAP (Ser127) antibody that can recognize the TAZ (Ser89) phosphorylation site because of sequence conservation (28). The images shown in the figures are representative of 3 independent experiments. The relative integrated density of each protein band was digitized using ImageJ (NIH).
Immunofluorescence. Primary murine osteoblasts or HEK293T cells grown on glass coverslips were fixed with $4 \%$ formaldehyde for 10 minutes, permeabilized, and incubated with the indicated antibodies (Figure 3, A and B, Figure 5A, and Supplemental Figure 3A). Following incubation with a secondary antibody, nuclei were stained with DAPI (Life Technologies, Thermo Fisher Scientific). Slides were mounted using Aqua-Mount Slide Mounting Media (Thermo Fisher Scientific). Confocal imaging was performed with an Olympus IX81 inverted microscope and FluoView software (Olympus).

Transient transfection and luciferase assay. To assess the modulation of the RUNX2-TAZ complex-driven gene expression by ABL, we used the BGLAP promoter-luciferase reporter construct (p6OSE2-luc) that contains 6 tandem repeats of the OSE2 element of the BGLAP promoter (52). To assess the modulation of PPAR $\gamma$-driven gene expression by ABL, we used the $a P 2$ promoter-containing luciferase reporter construct (aP2luc). HEK293T cells were transiently cotransfected with a p6OSE2-luc or aP2-luc reporter plasmid and a phRL-CMV plasmid (Promega), with or without RUNX2, TAZ, and ABL constructs, using LipoD293 DNA In Vitro Transfection Reagent (SignaGen Laboratories). Cells were lysed 24 hours after transfection and assayed for firefly and Renilla luciferase activity using the Dual-Luciferase Reporter System (Promega). The data are expressed as the ratio of firefly to Renilla activity.

BrdU assay. The BrdU incorporated into primary murine osteoblasts was measured with a BrdU Cell Proliferation ELISA Kit (Abcam) according to the manufacturer's protocol. Osteoblasts infected with shGFP or $\operatorname{sh} A B L$ were plated onto a 96-well plate and cultured for 6 hours. Then, cells were further incubated in BrdU-containing medium for 24 hours before adding the fixing solution.

Pulse-chase experiment. HEK293T cells were transiently cotransfected with ABL and TAZ constructs. Transfected cells were incubated in cysteine- and methionine-free DMEM complete medium for 30 minutes, then pulse-labeled with $\left[{ }^{35} \mathrm{~S}\right]$ methionine/cysteine for 2 hours. Labeling medium was then washed away and replaced with chase medium containing methionine and cysteine for the chase intervals as indicated in Figure 4D and Figure 6D as Chase time. ${ }^{35}$ S-labeled proteins were immunoprecipitated from cell lysates using the indicated antibodies in (Figure 4D and Figure 6D), separated by SDS-PAGE and analyzed by autoradiography as described previously (53).

In vivo ubiquitin assay. An in vivo ubiquitin assay was performed as described previously (54). Briefly, HEK293T cells were transiently cotransfected with expression vectors of HA-tagged ubiquitin (HA$\mathrm{Ub}$ ) in the presence or absence of ABL, TAZ, or SMURF1 constructs. Twenty-four hours after transfection, cells were treated with $10 \mathrm{mM}$ MG132 for four hours. Then, cells were lysed in complete cell lysis buffer (2\% SDS, $150 \mathrm{mM} \mathrm{NaCl}, 10 \mathrm{mM}$ Tris-HCl, $\mathrm{pH}$ 8.0) with protease and phosphatase inhibitors, boiled for 10 minutes, sheared with a sonication device, and incubated at $4^{\circ} \mathrm{C}$ for 30 minutes with dilution buffer (10 mM Tris-HCl, pH 8.0, 150 mM NaCl, 2 mM EDTA, 1\% Triton). After lysates were cleared by centrifugation for 30 minutes at $14,000 \mathrm{~g}$ and $4^{\circ} \mathrm{C}$, IP and Western blotting were performed with the indicated antibodies shown in Figure 4E, Figure 6E, Figure 7, B and D, and Supplemental Figure 7E as "IP."

Cell growth assays. MC3T3 cells infected with shGFP or $\operatorname{sh} A B L$, with or without a retroviral vector expressing TAZ (S89A), were replated in regular growth medium and placed into the INCUCYTE Kinetic Imaging System (Essen Bioscience) to monitor cell growth and the percentage of cell confluence as described previously (55). 
Statistics. All results are shown as the mean \pm SEM of data from at least 3 separate experiments. The data were subjected to ANOVA with Tukey-Kramer post-hoc test or an unpaired 2-tailed $t$ test with JMP 7 (SAS Institute Inc.) to determine differences. $P$ values of less than 0.05 were considered statistically significant.

Study approval. All animal studies were approved by the Animal Research Council of the University Health Network (Toronto, Ontario, Canada).

\section{Author contributions}

$\mathrm{YM}$ and RR designed the experiments. YM performed and analyzed the experiments. JLR, OAK, YS, JP, MV, JT, JRK, BY, MP, MFM, and MDG performed and analyzed specific experiments. JLR, ER, MN, ADL, MHO, AJK, GSF, JLW, and AMP contributed materials. YM and RR wrote the manuscript, with helpful comments from OAK, MJW, MN, and ADL.

\section{Acknowledgments}

This work was supported by grants from the Canadian Institute for Health Research (CIHR): 143420 Ontario Institute for Cancer Research (OICR): ITV; the NIH (R01CA155160 and R01CA195549); and the Spatio-Temporal Targeting and Amplification of Radiation Response (STTARR) program. RR is supported by the Princess Margaret Cancer Center and the Ontario Institute for Cancer Research. YM is supported by the Postdoctoral Fellowship for Research Abroad funded by the Japan Society for the Promotion of Science, the Japan Rheumatism Foundation, the Sumitomo Life Social Welfare Services Foundation, and the Nakayama Science Foundation.

Address correspondence to: Robert Rottapel, University Health Network TMDT site, 101 College Street, room 12-704, Toronto, Ontario, M5G 1L7, Canada. Phone: 416.581.7852; E-mail: rottapel@ uhnresearch.ca.
1. Hiromi Y, Gehring WJ. Regulation and function of the Drosophila segmentation gene fushi tarazu. Cell. 1987;50(6):963-974.

2. Serfling E. Autoregulation-a common property of eukaryotic transcription factors? Trends Genet. 1989;5(5):131-133.

3. Penn BH, Bergstrom DA, Dilworth FJ, Bengal E, Tapscott SJ. A MyoD-generated feed-forward circuit temporally patterns gene expression during skeletal muscle differentiation. Genes Dev. 2004;18(19):2348-2353.

4. Okuno $\mathrm{Y}$, et al. Potential autoregulation of transcription factor PU.1 by an upstream regulatory element. Mol Cell Biol. 2005;25(7):2832-2845.

5. Graf T, Enver T. Forcing cells to change lineages. Nature. 2009;462(7273):587-594.

6. Zhao B, et al. Inactivation of YAP oncoprotein by the Hippo pathway is involved in cell contact inhibition and tissue growth control. Genes Dev. 2007;21(21):2747-2761.

7. Zhao B, et al. Angiomotin is a novel Hippo pathway component that inhibits YAP oncoprotein. Genes Dev. 2011;25(1):51-63.

8. Zhang $\mathrm{H}$, et al. TEAD transcription factors mediate the function of TAZ in cell growth and epithelial-mesenchymal transition. J Biol Chem. 2009;284(20):13355-13362

9. Lai D, Ho KC, Hao Y, Yang X. Taxol resistance in breast cancer cells is mediated by the hippo pathway component TAZ and its downstream transcriptional targets Cyr61 and CTGF. Cancer Res. 2011;71(7):2728-2738.

10. Hong JH, et al. TAZ, a transcriptional modulator of mesenchymal stem cell differentiation. Science. 2005;309(5737):1074-1078

11. Ducy P, Zhang R, Geoffroy V, Ridall AL, Karsenty G. Osf2/Cbfa1: a transcriptional activator of osteoblast differentiation. Cell. 1997;89(5):747-754.

12. Komori $\mathrm{T}$, et al. Targeted disruption of $\mathrm{Cbfa} 1$ results in a complete lack of bone formation owing to maturational arrest of osteoblasts. Cell. 1997;89(5):755-764.

13. Kobayashi T, Kronenberg HM. Overview of skeletal development. Methods Mol Biol. 2014;1130:3-12.

14. Jones WA, Gerrie J, Pritchard J. Cherubismfamilial fibrous dysplasia of the jaws. J Bone Joint Surg Br. 1950;32-B(3):334-347.
15. Levaot N, et al. Loss of Tankyrase-mediated destruction of 3BP2 is the underlying pathogenic mechanism of cherubism. Cell. 2011;147(6):1324-1339.

16. Ueki $\mathrm{Y}$, et al. Mutations in the gene encoding c-Abl-binding protein SH3BP2 cause cherubism. Nat Genet. 2001;28(2):125-126.

17. Ren R, Mayer BJ, Cicchetti P, Baltimore D. Identification of a ten-amino acid proline-rich SH3 binding site. Science. 1993;259(5098):1157-1161.

18. Khatri A, Wang J, Pendergast AM. Multifunctional Abl kinases in health and disease. J Cell Sci. 2016;129(1):9-16.

19. Levaot N, et al. 3BP2-deficient mice are osteoporotic with impaired osteoblast and osteoclast functions. JClin Invest. 2011;121(8):3244-3257.

20. Li B, et al. Mice deficient in Abl are osteoporotic and have defects in osteoblast maturation. Nat Genet. 2000;24(3):304-308.

21. Jin $\mathrm{H}$, Wang JY. Abl tyrosine kinase promotes dorsal ruffles but restrains lamellipodia extension during cell spreading on fibronectin. Mol Biol Cell. 2007;18(10):4143-4154.

22. Mundlos S. Cleidocranial dysplasia: clini$\mathrm{cal}$ and molecular genetics. J Med Genet. 1999;36(3):177-182.

23. Barilá D, Superti-Furga G. An intramolecular SH3-domain interaction regulates c-Abl activity. Nat Genet. 1998;18(3):280-282.

24. Hantschel O, et al. Structural basis for the cytoskeletal association of Bcr-Abl/c-Abl. Mol Cell. 2005;19(4):461-473.

25. Levy D, Adamovich Y, Reuven N, Shaul Y. Yap1 phosphorylation by c-Abl is a critical step in selective activation of proapoptotic genes in response to DNA damage. Mol Cell. 2008;29(3):350-361.

26. Liu CY, et al. The hippo tumor pathway promotes TAZ degradation by phosphorylating a phosphodegron and recruiting the SCF $\{$ beta\}-TrCP E3 ligase. J Biol Chem. 2010;285(48):37159-37169.

27. Kanai F, et al. TAZ: a novel transcriptional co-activator regulated by interactions with 14-3-3 and PDZ domain proteins. ЕMBO J. 2000;19(24):6778-6791.

28. Lei QY, et al. TAZ promotes cell proliferation and epithelial-mesenchymal transition and is inhibited by the hippo pathway. Mol Cell Biol. 2008;28(7):2426-2436.

29. Liu CY, et al. PP1 cooperates with ASPP2 to dephosphorylate and activate TAZ. J Biol Chem. 2011;286(7):5558-5566.

30. Echarri A, Pendergast AM. Activated c-Abl is degraded by the ubiquitin-dependent proteasome pathway. Curr Biol. 2001;11(22):1759-1765.

31. Varelas X, et al. The Hippo pathway regulates Wnt/beta-catenin signaling. Dev Cell. 2010;18(4):579-591.

32. Yamashita M, et al. Ubiquitin ligase Smurf1 controls osteoblast activity and bone homeostasis by targeting MEKK2 for degradation. Cell. 2005;121(1):101-113.

33. Sprycel (dasatinib) [prescribing information]. Bristol-Myers Squibb Company. http:// packageinserts.bms.com/pi/pi_sprycel.pdf. Accessed October 25, 2016.

34. Cortes JE, Abruzzese E, Chelysheva E, Guha $\mathrm{M}$, Wallis N, Apperley JF. The impact of dasatinib on pregnancy outcomes. Am J Hematol. 2015;90(12):1111-1115.

35. Puri PL, Bhakta K, Wood LD, Costanzo A, Zhu J, Wang JY. A myogenic differentiation checkpoint activated by genotoxic stress. Nat Genet. 2002;32(4):585-593.

36. Innocenzi A, et al. An evolutionarily acquired genotoxic response discriminates $\mathrm{MyoD}$ from Myf5, and differentially regulates hypaxial and epaxial myogenesis. EMBO Rep. 2011;12(2):164-171.

37. Jang EJ, Jeong H, Han KH, Kwon HM, Hong JH, Hwang ES. TAZ suppresses NFAT5 activity through tyrosine phosphorylation. Mol Cell Biol. 2012;32(24):4925-4932.

38. Kua HY, et al. c-Abl promotes osteoblast expansion by differentially regulating canonical and non-canonical BMP pathways and p16INK4a expression. Nat Cell Biol. 2012;14(7):727-737.

39. Xing L, Zhang M, Chen D. Smurf control in bone cells. J Cell Biochem. 2010;110(3):554-563.

40. Kaneki $\mathrm{H}$, et al. Tumor necrosis factor promotes Runx2 degradation through up-regulation of Smurf1 and Smurf2 in osteoblasts. J Biol Chem. 2006;281(7):4326-4333.

41. Zhao M, Qiao M, Oyajobi BO, Mundy GR, Chen D. E3 ubiquitin ligase Smurf1 mediates core- 


\section{RESEARCH ARTICLE}

binding factor alpha1/Runx2 degradation and plays a specific role in osteoblast differentiation. J Biol Chem. 2003;278(30):27939-27944.

42. Shen R, et al. Smad6 interacts with Runx2 and mediates Smad ubiquitin regulatory factor 1-induced Runx2 degradation. J Biol Chem. 2006;281(6):3569-3576

43. Zhu H, Kavsak P, Abdollah S, Wrana JL, Thomsen GH. A SMAD ubiquitin ligase targets the BMP pathway and affects embryonic pattern formation. Nature. 1999;400(6745):687-693.

44. Zhao M, Qiao M, Harris SE, Oyajobi BO, Mundy GR, Chen D. Smurf1 inhibits osteoblast differentiation and bone formation in vitro and in vivo. J Biol Chem. 2004;279(13):12854-12859.

45. Chen $\mathrm{G}$, et al. The 3BP2 adapter protein is required for optimal B-cell activation and thymus-independent type 2 humoral response. Mol Cell Biol. 2007;27(8):3109-3122.
46. Narimatsu M, et al. Regulation of planar cell polarity by Smurf ubiquitin ligases. Cell. 2009;137(2):295-307.

47. Wetzel DM, McMahon-Pratt D, Koleske AJ. The Abl and Arg kinases mediate distinct modes of phagocytosis and are required for maximal Leishmania infection. Mol Cell Biol. 2012;32(15):3176-3186.

48. Zipfel PA, Grove M, Blackburn K, Fujimoto M, Tedder TF, Pendergast AM. The c-Abl tyrosine kinase is regulated downstream of the $\mathrm{B}$ cell antigen receptor and interacts with CD19. JImmunol. 2000;165(12):6872-6879.

49. Tybulewicz VL, Crawford CE, Jackson PK, Bronson RT, Mulligan RC. Neonatal lethality and lymphopenia in mice with a homozygous disruption of the c-abl proto-oncogene. Cell. 1991;65(7):1153-1163.

50. Ralston S, Helfrich MH, eds. Bone Research Protocols. New York, New York: Humana Press; 2003.
The Journal of Clinical Investigation

51. Zhao B, et al. TEAD mediates YAP-dependent gene induction and growth control. Genes Dev. 2008;22(14):1962-1971.

52. Ducy P, Karsenty G. Two distinct osteoblastspecific cis-acting elements control expression of a mouse osteocalcin gene. Mol Cell Biol. 1995;15(4):1858-1869.

53. Gong Y, et al. Nuclear export signal of androgen receptor (NESAR) regulation of androgen receptor level in human prostate cell lines via ubiquitination and proteasome-dependent degradation. Endocrinology. 2012;153(12):5716-5725.

54. Choo YS, Zhang Z. Detection of protein ubiquitination. JVis Exp. 2009;(30):1293.

55. Cullis J, et al. The RhoGEF GEF-H1 is required for oncogenic RAS signaling via KSR-1. Cancer Cell. 2014;25(2):181-195.

56. Nagy A. Cre recombinase: the universal reagent for genome tailoring. Genesis. 2000;26(2):99-109. 